\title{
Singular Bohr-Sommerfeld Rules
}

\author{
Yves Colin de Verdière ${ }^{1,2}$, Bernard Parisse ${ }^{2}$ \\ 1 Institut Universitaire de France \\ 2 Institut Fourier, Unité mixte de recherche, CNRS-UJF 5582, BP 74, 38402 Saint Martin d'Hères Cedex, \\ France. E-mail: yves.colin-de-verdiere@ujf-grenoble.fr; bernard.parisse@ujf-grenoble.fr
}

Received: 22 October 1998 / Accepted: 10 March 1999

\begin{abstract}
The object of this paper is to present a geometrical formulation of the singular Bohr-Sommerfeld rules given in [8], in a more general context, including characteristic manifolds with several saddle points. Several examples are detailed and numerical checkings of the results are provided.
\end{abstract}

\section{Introduction}

Bohr-Sommerfeld rules allow to describe the semi-classical behavior of eigenvalues for a completely integrable Hamiltonian system; in fact, they are only concerned with the open dense subset of the phase space foliated by invariant Lagrangian tori and are expressed as quantization rules which select some discrete set of these tori (see $[3,13]$ or [20]). These rules make use of action integrals and integrals of the sub-principal symbol along the cycles of the invariant torus. It is possible to reinterpret these rules outside any spectral context as existence conditions for solutions of a pseudo-differential equation for which this torus is a Lagrangian submanifold of the characteristic manifold. In dimension 1 , if $\gamma$ is a smooth compact connected component of the characteristic manifold ( $\gamma$ is a topological circle), let us denote by $A_{\gamma}=\int_{\gamma} \xi d x$ the action integral and by $I_{\gamma}=\int_{\gamma} H_{1} d t$ ( $H_{1}$ is the sub-principal symbol). If $\mathcal{X}_{H_{0}}$ is the Hamiltonian vector field associated with the principal symbol $H_{0}, d t$ is defined by $d t\left(\mathcal{X}_{H_{0}}\right)=1$ and $m(\gamma)$ is the Maslov index. A necessary and sufficient condition for the existence of a function $u$ satisfying $\hat{H} u=O\left(h^{2}\right)$ with $L^{2}$ norm bounded from below, can be written as follows:

$$
\frac{1}{h} A_{\gamma}-I_{\gamma}+\frac{\pi}{2} m(\gamma) \in 2 \pi \mathbb{Z}
$$

In dimension 1 already, a generic Hamiltonian $H_{0}(x, \xi)$ admits, in general, critical values with singular corresponding level sets. Near some local non degenerate extremum, it is always possible to regularize the equations using harmonic oscillators (see [4]). This 
is no more true for saddle points. There exists, however, a microlocal normal form (semiclassical Morse lemma, see [7,6]). In the paper [8], we described the quantization rules associated with the singular manifold in the case of a double potential well for the Schrödinger equation.

The aim of the present paper is to reformulate and extend the quantization rules of [8]. The reformulation is purely geometrical: the action integrals do not need to be regularized, but the integrals of the sub-principal symbol are divergent and need to be regularized using the symplectic geometry of the phase space (see 2.2).

In such a way, it becomes possible to describe the semi-classical spectrum in cases where the characteristic manifold do admit several saddle points: multiple wells with saddle at the same energy, pendulum on the torus. The case of coupled systems (see the local theory in [6]) will be described in [5].

It is also possible to study scattering problems. The case of a potential with one threshold only and the volcano-top are presented (see Sect. 13). In [5], we study the adiabatic limit for a $N$-levels system with avoided eigenvalues crossings (see [18] and also [16]). For comparison with existing texts, we insist on the fact that our methods are situated in the smooth context and do not use analytic extensions. Subtle combinatorics of Stokes lines (see [12] and [11]) is replaced by some algebraic mechanism on the characteristic manifold which is a tetravalent graph.

The paper is organized as follows: microlocal solutions of the pseudo-differential equation are sections of a sheaf on the characteristic manifold $Z$. The abstract description of these sheafs is provided in Sect. 4. The geometric description $\left(\bmod O\left(h^{2}\right)\right)$ of this sheaf is given in terms of the classical invariants of cycles (Sect. 2) and saddle points (Sect. 3). We consider in particular some canonical regularization of the integrals $\int_{\gamma} H_{1} d t$, where $\gamma$ is a cycle of the characteristic manifold.

After that, we show how to perform the computations on several examples: eigenvalues of the Schrödinger operator with a double well on $\mathbb{R}$ in order to recover results of [8] (Sect. 8), with a triple well (Sect. 9), discriminant and spectra of Hill's equation (Sects. 10 and 11), system of two Schrödinger operators with a small coupling parameter (Sect. 12), computation of the scattering matrix for potential on the real line with 1 (Sect. 13.1) or 2 channels (Sect. 13.2).

At the end, in the appendices (Sect. A), the theoretical results are compared with direct numerical computations.

The cases of systems of Schrödinger equations (Born-Oppenheimer approximation) and of the adiabatic limit for avoided crossings for an $N$-levels system will be described in [5].

The case of dimensions $\geq 2$ will be studied in some future work with application to semi-excited states in the resonant $2 d$ case (see [17] and [2]). Focus-focus singularities are discussed in [24] and [25]. General Morse-Bott singularities in dimension 2 will be described in [10].

\section{The Context}

1.1. Classics. Let $(X, \omega)$ be a 2-dimensional symplectic manifold ( $X$ is the phase space) and $H_{0}: X \rightarrow \mathbb{R}$ a smooth Hamiltonian. In this paper, $X$ is the cotangent bundle of $M=\mathbb{R}$ or of $M=\mathbb{R} / \mathbb{Z}$, but it is also possible to work with a Riemann surface $X$ (Toeplitz operators).

Let us assume that 0 is a critical value of $H_{0}$ and let $Z$ be some connected component of the characteristic manifold $H_{0}^{-1}(0)$. Let us assume that all critical points of $H_{0}$ located 


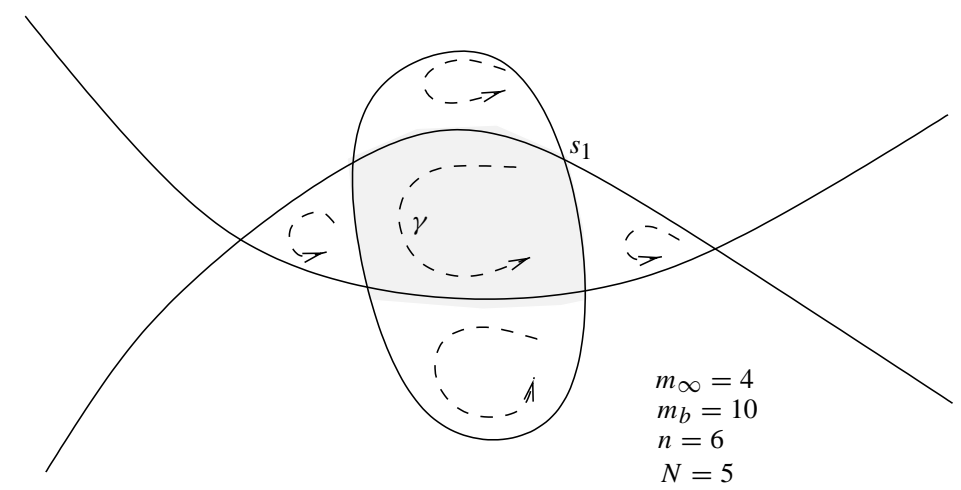

Fig. 1. The characteristic manifold $Z$

on $Z$ are non degenerated saddle points. For eigenvalues problems, $Z$ is a compact set, while $Z$ admits some open edges for the scattering problems. Let us denote by $m_{\infty}$ the number of open edges of $Z, Z_{0}$ the set of all critical points and $n=\# Z_{0}$. The set $Z$ is a tetravalent graph embedded in $X$. We will denote by $s_{j}, j=1, \cdots, n$ the points of $Z_{0}$ (vertices of $Z$ ), $e_{l}, l=1, \cdots, m$ the edges of $Z$ (compact or open), let us define $m=m_{b}+m_{\infty}$. Let $\gamma_{j}, j=1, \cdots, N$ be a basis of cycles of $Z$, i.e. a basis of $\mathbf{H}_{1}(Z, \mathbb{Z})$ (free $\mathbb{Z}-$ module of rank $N$ ) which consists of geometric oriented cycles. Euler-Poincaré formula implies:

$$
N=b_{1}(Z)=1-n+m_{b},
$$

with $4 n=2 m-m_{\infty}$. In the compact case $N=1+n$.

1.2. Microfunctions. In this section we will give precise definitions and notations concerning microlocalization (useful references are [7,22] and [25]). We want to study the solutions $u$ of a differential equation of order $m, \hat{H} u=0$, on the real line, depending on the small real parameter $h$. The general form of $\hat{H}$ is

$$
\hat{H}=\sum_{\alpha=0}^{m} a_{\alpha}(x, h) D_{x}^{\alpha},
$$

where $D_{x}=\frac{h}{i} \frac{d}{d x}$ and the $a_{\alpha}(x, h)$ 's are classical smooth symbols

$$
a_{\alpha}(x, h) \sim \sum_{j=0}^{\infty} a_{\alpha, j}(x) h^{j} .
$$

We will always assume that

$$
H_{0}(x, \xi)=\sum_{\alpha=0}^{m} a_{\alpha, 0}(x) \xi^{\alpha}
$$

is real valued. $H_{0}$ is the principal symbol or classical Hamiltonian associated with $\hat{H}$. Our goal is to study solutions of $\hat{H} u=O\left(h^{\infty}\right)$ in the $h \rightarrow 0$ limit (semi-classical limit) by using microlocal analysis: it means first studying solutions locally in the phase space $X=T^{\star} \mathbb{R}$ and then gluing the pieces together. 
Definition 1. $\left.\left.u_{h}(x), h \in\right] 0, h_{0}\right]$, a family of distributions $\left(u_{h}(x) \in \mathcal{D}^{\prime}(\mathbb{R})\right)$, is an admissible functional if, for any $\chi \in C_{o}^{\infty}(\mathbb{R})$, there exist $s$ and $N$ such that

$$
\left\|\chi u_{h}\right\|_{s}=O\left(h^{-N}\right),
$$

where $\|.\|_{s}$ is the Sobolev norm. For example an h-independent distribution is an admissible functional. Let us denote by $\mathbb{C}_{h}$ the quotient ring of (germs of) functions $\left.\lambda:] 0, h_{0}\right] \rightarrow \mathbb{C}$ which satisfy $\lambda(h)=O\left(h^{-N}\right)$ for some $N$ by the ideal of $\lambda$ 's which satisfy $\lambda(h)=O\left(h^{\infty}\right)$ (the field of formal Laurent series in $h$ is a subring of $\mathbb{C}_{h}$ ).

We can also define negligible functionals $u_{h}$ by

$$
\forall s, N, \chi,\left\|\chi u_{h}\right\|_{s}=O\left(h^{N}\right)
$$

and the microsupport $W F_{h}\left(u_{h}\right)$ by

$$
\left(x_{0}, \xi_{0}\right) \notin W F_{h}\left(u_{h}\right)
$$

if and only if there exists $\chi \in C_{o}^{\infty}$ such that $\mathcal{F}\left(\chi u_{h}\right)(\xi)=O\left(h^{\infty}\right)$ in some fixed neighbourhood of $\xi_{0}$.

Here $\mathcal{F}$ is the $h$-Fourier transform defined by

$$
\mathcal{F} u(\xi)=(2 \pi h)^{-\frac{1}{2}} \int_{\mathbb{R}} e^{-i x \xi / h} u(x) d x .
$$

Important remark. $W F_{h}\left(u_{h}\right)=\emptyset$ does not imply that $u_{h}$ is negligible. $u_{h}(x)=e^{i x / h^{2}}$ is an example. But, if we know moreover that $u_{h}$ is a solution of $\hat{H} u_{h}=0$, where $H_{0}(x, \xi)=a_{m, 0}(x) \xi^{m}+\cdots$ with $a_{m, 0}$ non vanishing, then it is true by usual ellipticity.

The most appropriate language for what follows is to speak of sheaves (see [26]). We will define the sheaf $\mathcal{M}_{X}$ on $X=T^{\star} \mathbb{R}$ of microfunctions on $X$ on which the sheaf of pseudo-differential operators is acting. We will view it as a sheaf of modules on the ring $\mathbb{C}_{h}$. If $U \subset X$ is an open set, the module $\mathcal{M}_{X}(U)$ of sections of $\mathcal{M}_{X}$ on the open set $U$, is the quotient of admissible functionals $u_{h}$ by the equivalence relation $\left[u_{h} \sim v_{h}\right.$ if and only if $\left.W F_{h}\left(u_{h}-v_{h}\right) \cap U=\emptyset\right]$. We will call $\mathcal{M}_{X}(U)$ the set of microfunctions on $U$. We will write $u_{h}=O\left(h^{\infty}\right)$ near $z_{0}$ as a way to say that $z_{0} \notin W F_{h}\left(u_{h}\right)$.

Solutions of $\hat{H} u=O\left(h^{\infty}\right)$ can be studied by introducing the subsheaf $\mathcal{K}_{\hat{H}}$ of $\mathcal{M}_{X}$ which is the kernel of $\hat{H}$ (microlocal solutions of $\hat{H}$ ) whose support is $Z=H_{0}^{-1}(0)$ and solutions of $\hat{H} u=O\left(h^{\infty}\right)$ are global sections of $\mathcal{K}_{\hat{H}}$.

A typical example of equation is

$$
\hat{H}=\hat{P}-E_{0}-E h,
$$

where $\hat{P}$ is a fixed formally selfadjoint operator and $\mathrm{E}$ is a parameter which stays in a compact domain of $\mathbb{C}$ and with respect to which all estimates are uniform. Schrödinger operators

$$
\hat{P}=-\frac{h^{2}}{2} \frac{d^{2}}{d x^{2}}+V(x)-E_{0}-E h,
$$


where $V$ is a smooth real valued function, are the most usual examples.

If $E$ is real, solutions of $\hat{H} u=O\left(h^{\infty}\right)$ correspond to eigenfunctions of $\hat{P}$ with eigenvalue $E_{0}+h E$. In this example $H_{0}=\frac{1}{2} \xi^{2}+V(x)-E_{0}$.

We can also define the sub-principal symbol $H_{1}$ of $\hat{H}$ by using the Weyl quantization

$$
\hat{H}=O p_{W}\left(H_{0}+h H_{1}\right)+\hat{Q}_{2},
$$

where $Q$ has degree 2 with respect to $h$, meaning that $a_{\alpha}(x, h)=O\left(h^{2}\right)$. In example 3, $H_{1}=-E$. The Weyl quantization, $O p_{W}$, is defined by

$$
\mathrm{Op}_{\mathrm{W}}(H) u(x)=\frac{1}{2 \pi h} \int e^{e^{\frac{i}{h}(x-y) \xi}} H\left(\frac{x+y}{2}, \xi\right) u(y)|d y d \xi| .
$$

1.3. Spectrum. Assume that $\hat{H}$ is formally symmetric on $C_{o}^{\infty}(\mathbb{R}) \subset L^{2}(\mathbb{R}, d x)$ and let us denote by $\hat{H}_{a}$ any self-adjoint extension of $\hat{H}$ whose spectrum is denoted by $\sigma\left(\hat{H}_{a}\right)$. Let us suppose that $Z$ is compact, then if we can find non-negligible solutions $u$ of $\hat{H} u=O\left(h^{\infty}\right)$ (with compact support) it implies that distance $\left(0, \sigma\left(\hat{H}_{a}\right)\right)=O\left(h^{\infty}\right)$ by the minimax principle.

Singular Bohr-Sommerfeld rules are geometric rules which are equivalent to the existence of functions $u$ which satisfies

$$
\hat{H} u=O\left(h^{2}\right),
$$

are microlocalized on $Z$ and satisfy the lower bound $\int|u(x)|^{2} d x \geq 1$. In fact we will prove existence of non-trivial functions $u$ which satifies $\hat{H} u=O\left(h^{\infty}\right)$ but, in Example 2, geometric conditions will be computed only to the first non-trivial order and determine $E_{0}+E h$ up to $O\left(h^{2}\right)$.

1.4. Regular Bohr-Sommerfeld rules. We can reformulate the usual Bohr-Sommerfeld rules using the previous definitions.

1.4.1. WKB solutions. Assume $Z=H_{0}^{-1}(0)$ is compact and smooth. Then the sheaf $\mathcal{K}_{\hat{H}}$ is locally free of rank one on $Z$. We will call $W K B$-solution near $z_{0} \in Z$ a generator of the free module $\mathcal{K}_{\hat{H}}(U)$, where $U$ is a small neighbourhood of $z_{0}$. Any WKB solution can be written outside the caustic in the following form:

$$
u_{h}(x)=\left(\sum_{j=0}^{\infty} a_{j}(x) h^{j}\right) e^{\frac{i}{h} S(x)-i \int_{x_{0}}^{x} H_{1} d t} .
$$

If $\pi: X \rightarrow \mathbb{R}$ is the canonical projection, the half-density $\sigma_{0}=\pi^{\star}\left(a_{0}(x)|d x|^{\frac{1}{2}}\right)$ is invariant by the Hamiltonian flow on $Z=\left\{\left(x, S^{\prime}(x)\right)\right\}$. Remark that the principal symbol of $u_{h}$ (after the definition of [13]) is $\sigma_{0} e^{\Im\left(\int_{x_{0}}^{x} H_{1} d t\right)}$ which is $\sigma_{0}$ if $H_{1}$ is real valued. We can choose a normalization (depending on $x_{0}$ if $H_{1}$ is not real valued) such that $\sigma_{0}=|d t|^{\frac{1}{2}}$. We will say then that $u_{h}(x)$ is a normalized WKB solution of $\hat{H} u=O\left(h^{\infty}\right)$ at the point $x=x_{0}$.

It is the result of efforts of many people like Maslov and Hörmander that it is possible to define also WKB solutions near the caustic points. 
1.4.2. Holonomies and Bohr-Sommerfeld rules. For any oriented connected component $C$ of $Z$ (a topological circle) we can define the holonomy hol $(C)$ of the restriction to $C$ of this sheaf.

$$
\operatorname{hol}(C)=e^{\frac{i}{h} \alpha(h)}
$$

where $\alpha(h)$ is a formal series in $h$

$$
\alpha(h) \sim \sum_{j=0}^{\infty} \alpha_{j} h^{j}
$$

with $\alpha_{0}=\int_{C} \xi d x$ and $\alpha_{1}=-\int_{C} H_{1} d t+\frac{\pi}{2} m(C)$, where $m(C)$ is the Maslov index. Writing hol $(C)=1$ gives a necessary and sufficient condition for the existence of nonzero solutions microlocalized on $C$ of $\hat{H} u=O\left(h^{\infty}\right)$. Using the main example (Eq. (2)), we get then implicit relations for $E$ which describe the spectrum $\bmod O\left(h^{\infty}\right)$ in the interval $\left[E_{0}-M h, E_{0}+M h\right]$.

Theorem 1. Let $a_{C}(h)$ be a function which admits the formal series $\alpha(h)$ as Taylor expansion at $h=0$. If $Z$ is compact and smooth, there is, for $h$ small enough, a bijection between eigenvalues in $\left[E_{0}-M h, E_{0}+M h\right]$ and the solutions of $e^{i a_{C}(h) / h}=1$ for all components $C$ of $Z$ which associate eigenvalues and solutions which are at a distance $O\left(h^{\infty}\right)$.

In one direction, one uses the minimax principle. In the other one uses the fact that the sheaf is locally of dimension 1 .

1.5. $\mathcal{S}$ matrix. Let us assume in this section that $\hat{H}$ is formally self-adjoint and that $Z$ admits $m_{\infty}=2 \mu_{\infty}$ open edges. Let $D$ be a compact domain with boundary $\partial D$ in $X$ which contains the compact edges of $Z$ in his interior and such that $\partial D$ intersects transversally each open edge at 1 point. Each edge is oriented by the Hamiltonian flow of $H_{0}$. There are as many out-going edges as in-going edges, they alternate when we turn around $\partial D$, because domains where $H_{0}>0$ and $H_{0}<0$ alternate. We assume that the $e_{i}$ 's are in-going for $i=1, \cdots, \mu_{\infty}$ and out-going for $i=\mu_{\infty}+1, \cdots, m_{\infty}$.

Let $\Omega$ be a neighborhood of $\partial D$ in $X$ which intersects only open edges and $U=$ $D \cup \Omega$. We define $\mathcal{H}_{i}=\mathcal{K}_{\hat{H}}\left(\Omega \cap e_{i}\right.$ ) (free module of rank 1 over $\left.\mathbb{C}_{h}\right), \mathcal{H}_{i n}=\oplus_{i=1}^{\mu} \mathcal{H}_{i}$, $\mathcal{H}_{\text {out }}=\bigoplus_{i=\mu_{\infty}+1}^{m_{\infty}} \mathcal{H}_{i}$ and $\mathcal{H}_{\infty}=\mathcal{H}_{\text {in }} \oplus \mathcal{H}_{\text {out }}$.

Definition 2. We call the $\mathcal{S}$-matrix of $\hat{H}$ the subspace of $\mathcal{H}_{\infty}$ defined as the restrictions from functions of $\mathcal{K}_{\hat{H}}(Z)$ to $\mathcal{H}_{\infty}$, i.e. the sets of microlocal solutions of $\hat{H} u=0$ on open edges which admit an extension as solutions on the whole set Z. This space is in general the graph of a linear mapping, also denoted by $\mathcal{S}$ from $\mathcal{H}_{\text {in }}$ into $\mathcal{H}_{\text {out }}$.

We want to define the currents as an Hermitian form $J$ on $\mathcal{H}_{\infty}$.

Let $\Pi$ be a self-adjoint PDO of order 0 whose microsupport is contained in $U$ and which is microlocally equal to Id in some neighborhood of $D \backslash \Omega$. Let $\Sigma$ be another self-adjoint PDO which vanishes microlocally in some other neighborhood of $D \backslash \Omega$ and which coincides with Id where $\Pi$ does not.

Definition 3. We define currents as the Hermitian form

$$
J(u, v)=\frac{i}{h}(<\Sigma \hat{H} \Pi u|\Pi v>-<\Pi u| \Sigma \hat{H} \Pi v>) .
$$




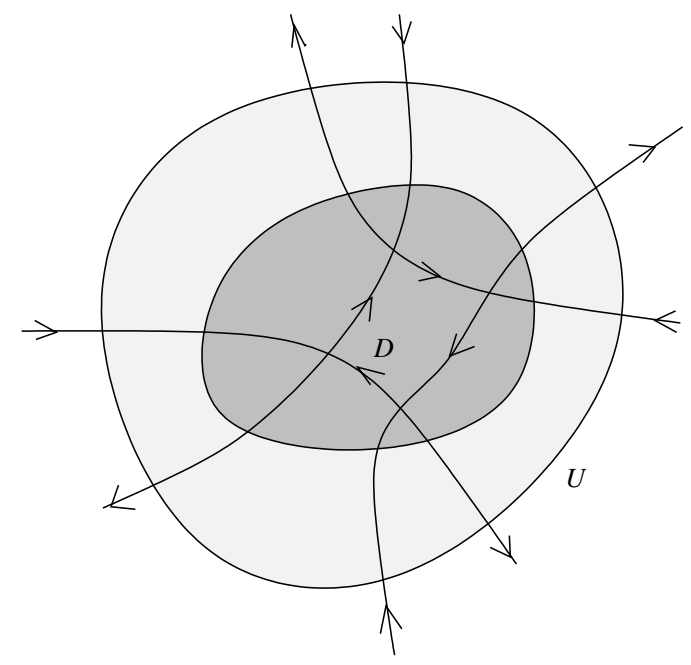

Fig. 2. $\mathcal{S}$ matrix

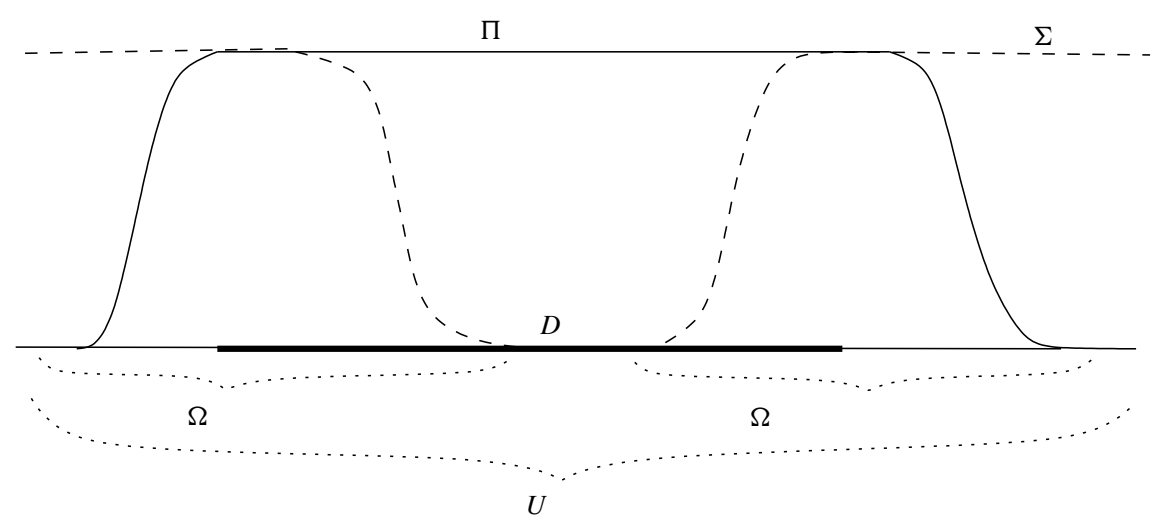

Fig. 3. How to define $J$

It is possible to compute $J(u, u)$ for any microlocal solution $u$ on the edge $e$ in terms of his principal symbol $\sigma_{0}(u)$ which is an half-density on $e$ invariant by the Hamiltonian flow of $H_{0}$.

\section{Lemma 1.}

$$
J(u, u)= \pm \frac{\left|\sigma_{0}\right|^{2}}{|d t|}+O(h),
$$

with the + sign for an out-going edge and the - sign for an in-going edge. Here $\left|\sigma_{0}\right|^{2} / d t$ denotes the quotient of a density (square of an half-density) by $d t$ and not a derivative.

Proof. In fact, we have:

$$
J(u, v)=\frac{i}{h}<[\Sigma, \hat{H}] \Pi u\left|\Pi v>=\frac{i}{h}<[\Sigma, \hat{H}] u\right| v>+O\left(h^{\infty}\right) .
$$


If $u$ is a microlocal (WKB) solution on $e_{i}$, we denote by $\sigma_{0} \in \Omega^{\frac{1}{2}}\left(e_{i}\right)$ his principal symbol and we have for any PDO $\hat{A}$ whose symbol vanishes outside $\Omega$ :

$$
<\left.\hat{A} u\left|u>=\int_{e_{i}} A_{0}\right| \sigma_{0}\right|^{2}+O(h),
$$

and the lemma follows from the computation of the symbol of the bracket $[\Sigma, \hat{H}]$ which is $\frac{h}{i} \frac{d \sigma}{d t}$ if we denote by $\sigma$ the principal symbol of $\Sigma$. We have then

$$
J(u, u)=\int_{e_{i}} \frac{d \sigma}{d t}\left|\sigma_{0}\right|^{2}+O(h),
$$

which gives the result because $\frac{\left|\sigma_{0}\right|^{2}}{d t}$ is time independent for a self-adjoint $\hat{H}$.

The microlocal solutions on different open edges are $J$-orthogonals (by the fact that PDO's reduce the WF) and, by Lemma 1, the form $J$ is positive (resp. negative) definite on the out-going (resp. in-going) edges.

The following result is not useful for what follows. The sketch of the proof is postponed to Appendix B.

Theorem 2. The $\mathcal{S}$-matrix is the graph of an unitary mapping from $\mathcal{H}_{\text {in }}$ to $\mathcal{H}_{\text {out }}$ with respect to the metrics induced respectively by $J$ on $\mathcal{H}_{\text {out }}$ and $-J$ on $\mathcal{H}_{\text {in }}$.

\section{Semi-Classical Invariants of Cycles}

To each cycle $\gamma$ of $Z$, we associate 3 numbers: the principal action $A_{\gamma}$ (more precisely $\left.\exp \left(\frac{i}{h} A_{\gamma}\right)\right)$, the sub-principal action $I_{\gamma}$ and the Maslov index $m(\gamma)$.

2.1. Principal actions $A_{\gamma}$. If $\gamma$ is a cycle of $Z$, we put:

$$
A_{\gamma}=\int_{\gamma} \xi d x
$$

If $\gamma=\partial D$ is the boundary of a compact domain $D$ of the phase space, the number $A_{\gamma}$ is the symplectic area of the $D$.

2.2. Sub-principal actions $I_{\gamma}$. If $\mathcal{X}_{H_{0}}$ is the symplectic gradient of $H_{0}$, we denote by $d t$ the associated differential form on $Z \backslash Z_{0}$ defined by:

$$
d t\left(\mathcal{X}_{H_{0}}\right)=1
$$

The numbers $I_{\gamma}$ are principal parts of the divergent integrals $\int_{\gamma} H_{1} d t$. It is enough to describe how principal parts should be derived near saddle points.

Let $A B$ be an arc of $\gamma$ on which the unique singularity is $s$. We define

$$
\mathrm{vp} \int_{A}^{B} H_{1} d t=\lim _{a, b \rightarrow 0}\left(\int_{A}^{a} H_{1} d t+\int_{b}^{B} H_{1} d t+a\left(s_{j}\right) \ln \left|\int_{R_{a, b}} \omega\right|\right),
$$




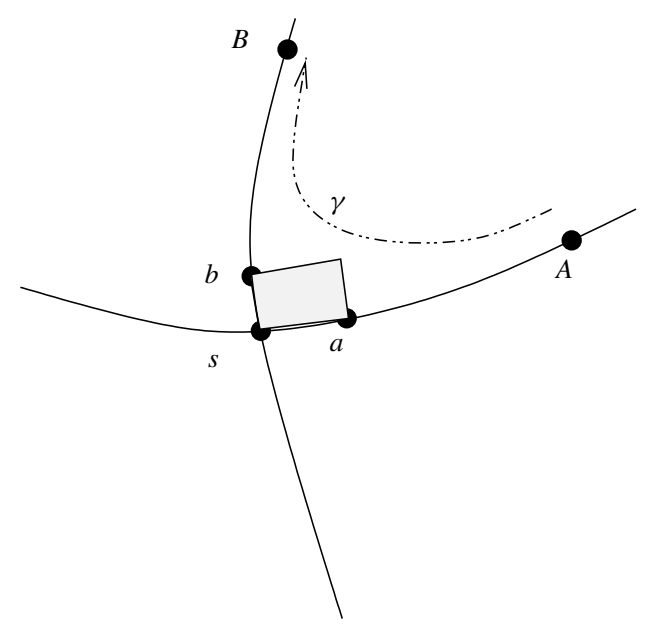

Fig. 4. Computation of the principal part of $\int_{\gamma} H_{1} d t$

where

$$
a\left(s_{j}\right)=\frac{ \pm H_{1}\left(s_{j}\right)}{\left|\operatorname{det} H_{0} "\left(s_{j}\right)\right|^{1 / 2}},
$$

and $R_{a, b}$ is the parallelogram built on the vectors $\boldsymbol{s}_{j} \boldsymbol{a}$ and $\boldsymbol{s}_{\boldsymbol{j}} \boldsymbol{b}$ drawn on the figure. The sign \pm is + if $A B$ is oriented in the same direction as $d t$ and - otherwise.

Lemma 2. The previous limit exists and is independent of the choices.

Proof. Performing first a canonical transformation, we may assume that $H_{0}(z)=W x \xi+$ $O\left(|z|^{3}\right)$. Of course, we have $W= \pm\left|\operatorname{det} H^{\prime \prime}{ }_{0}\left(s_{j}\right)\right|^{1 / 2}$ with the same sign convention as in formula (8). We can then compute $\left(\int_{A}^{a}+\int_{b}^{B}\right) H_{1} d t$ by replacing $H_{0}$ by $W x \xi$ (then $d t=\frac{d x}{W x}=-\frac{d \xi}{W \xi}$ ), which gives the following divergent part of the integral: $-H_{1}\left(s_{j}\right) / W \ln (a b)$.

If the cycle $\gamma$ is smooth at $s_{j}$, we define

$$
\tilde{\int}_{B}^{A} H_{1} d t=\lim _{\varepsilon \rightarrow 0^{+}}\left(\int_{B}^{\gamma(-\varepsilon)}+\int_{\gamma(\varepsilon)}^{A}\right) H_{1} d t
$$

where $u \rightarrow \gamma(u)$ is a $C^{1}$ parameterization of $\gamma$ and with $\gamma(0)=s_{j}$. This choice is compatible with the previous ones: $\gamma \rightarrow I_{\gamma}$ is a $\mathbb{Z}$-linear mapping.

Remark 1. It is possible to check that the map $\gamma \rightarrow I_{\gamma}$ which is at the moment only defined for cycles of $Z$ which have corners (angles $<\pi$ ) extends (uniquely) by $\mathbb{Z}-$ linearity to $H_{1}(Z, \mathbb{Z})$.

Remark 2. Sub-principal actions $I_{\gamma}$ do admit a natural interpretation in symplectic geometry. Let us assume that $\gamma$ is a simple cycle of $Z=\left\{H_{0}=0\right\}$ such that $\gamma=\partial D$ and that $H_{0}$ is $\leq 0$ inside $D$. Denote by $s_{j}, j=1, \cdots N$ the saddle points of $Z$ located on $\gamma$. Let $H_{1}: X \rightarrow \mathbb{R}$ be such that $\forall j, 1 \leq j \leq N$, we have $H_{1}\left(s_{j}\right)<0$. For $\tau<0$, let 


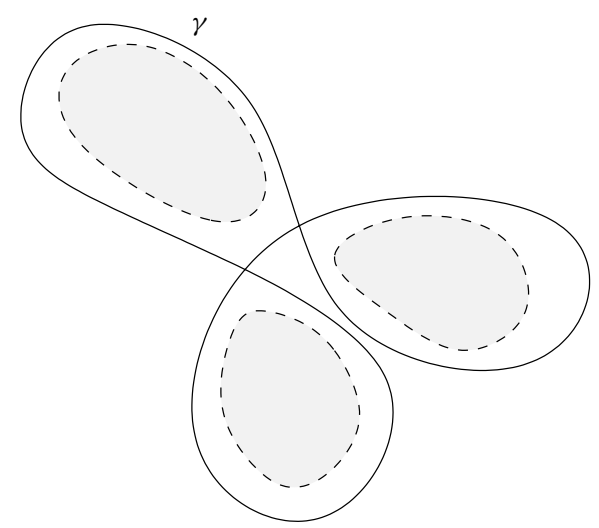

Fig. 5. Area $A(\tau)$

us define

$$
A(\tau)=\int_{H_{0}+\tau H_{1} \leq 0}|d x d \xi|
$$

Then, if $\tau \rightarrow 0^{-}, A(\tau)$ admits the following expansion:

$$
A(\tau)=A(0)+\tau\left(\sum_{j=1}^{N} \varepsilon_{0}\left(s_{j}\right)\left(1-\ln \left|\tau \varepsilon_{0}\left(s_{j}\right)\right|\right)\right)-\tau I_{\gamma}+O\left(\tau^{2} \ln |\tau|\right)
$$

2.3. Maslov indices $m(\gamma)$. We define the Maslov index of a smooth loop $\gamma$ following the usual recipe which is summarized on Fig. 6.

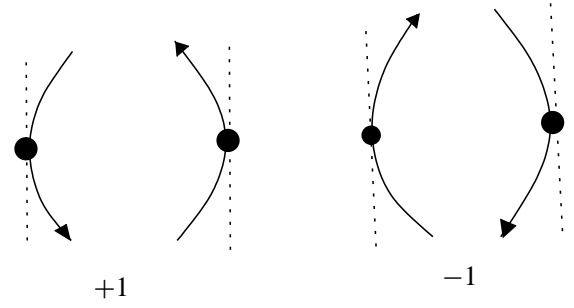

Fig. 6. Recipes for Maslov indices

Assuming that the tangent vectors to edges at singular points of the singular loop $\gamma$ are non vertical (i.e. non-parallel to the $\xi$ axis), we take the same definition counting with \pm 1 the caustics points of the non-singular part of the loop. If a tangent vector to $Z$ at a singular point is vertical, we make a small move of $Z$ in order to remove it: there are essentially 2 ways to do that. We choose one and keep the result in memory for the labeling of edges (see Fig. 8) coming at these singular points following Fig. 7. 


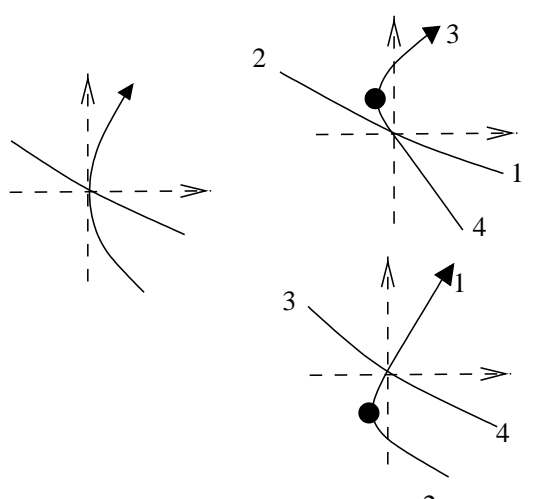

Fig. 7. Desingularizations

\section{Semi-Classical Invariants of the Saddle Points}

3.1. Normal form. We may reformulate the semi-classical Morse lemma (see [7, pp. 1546f.] and [6]) in the following way:

Theorem 3. There exists a (germ of) canonical transformation $\chi$ defined on $\left(\mathbb{R}^{2}, O\right)$, $(x, \xi)=\chi(y, \eta)$ which satisfy $H_{0} \circ \chi=W(y \eta) y \eta$ and $\chi(O)=s, s \in Z_{0}$. Then there exists an elliptic FIO $\hat{U}$ associated with $\chi$, a PDO $\hat{W}$, elliptic at the point $O$, and a unique formal series

$$
\varepsilon(h) \sim \sum_{j=0}^{\infty} \varepsilon_{j} h^{j}
$$

such that:

$$
\hat{H} \hat{U}=\hat{U} \hat{W}(\hat{y \eta}-h \varepsilon(h)),
$$

microlocally near $O$.

$$
\hat{y \eta}=\frac{h}{i}\left(y \frac{d}{d y}+\frac{1}{2}\right)
$$

is the Weyl quantization of $y \eta$. We have:

$$
\varepsilon_{0}= \pm \frac{H_{1}(s)}{\left|\operatorname{det}\left(H_{0}^{\prime \prime}(s)\right)\right|^{1 / 2}} .
$$

The sign \pm is the opposite of that of $W(0)$.

Proof. A very similar theorem is proved in [6] or in [7]. For completeness, we will give a simpler proof here not using the Morse lemma of [9].

- We first prove the existence of $\chi$. We may assume that $s=O$ and both branches of $Z$ near $s$ are tangent respectively to the axes $\{\eta=0\}$ and $\{y=0\}$ by an affine canonical transformation. It is now enough to use canonical transformations $\chi_{1}(y, \eta)=(y, \eta-$ $a(y))$ and $\chi_{2}(y, \eta)=(y-b(\eta), \eta)$ with appropriate functions $a$ and $b$ in order that the branches of $Z$ near $O$ are the axes. We get then a function $\psi(y, \eta)$ vanishing on the axis which can be divided by $y \eta$ inside smooth functions. 

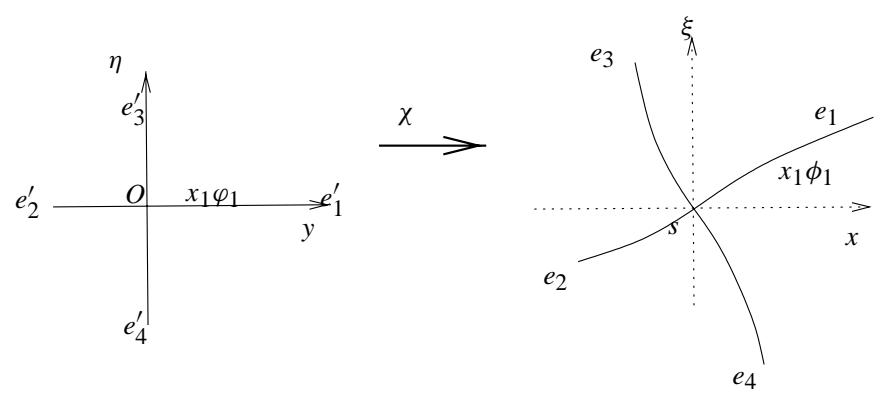

Fig. 8. The choices of the $e_{i}$ 's and of $\chi$

- If $\hat{U}_{0}$ is any FIO associated with $\chi$ we get by Egorov's theorem

$$
\hat{U}_{0}^{-1} \hat{H} \hat{U}_{0}=\hat{W}_{0}\left(\hat{y \eta}+h \hat{P}_{1}\right)
$$

where $\hat{W}_{0}$ and $\hat{P}_{1}$ are PDO's of order 0 . The first step is to find $\hat{\Pi}$ and $\hat{R}_{1}$, PDO's of order 0 , such that

$$
\hat{\Pi}^{-1}\left(\hat{y \eta}+h \hat{P}_{1}\right) \hat{\Pi}=\left(\operatorname{Id}+h \hat{R}_{1}\right)\left(\hat{y \eta}+\varepsilon_{0} h+h^{2} \hat{P}_{2}\right)
$$

where $\hat{P}_{2}$ is of order 0 . Denoting by the same small letters the principal symbols we get

$$
\frac{1}{i \pi}\{y \eta, \pi\}=\varepsilon_{0}+r_{1} y \eta-p_{1} .
$$

This equation can be solved in $\pi$ if and only if the Taylor expansion of the righthand side does not contain powers of $y \eta$ (see [9, p. 286] or [15, p. 175]) which can be achieved by appropriate choices of $\varepsilon_{0}$ and $r_{1}$. This is the first step of a proof by induction. Next steps are similar.

- Uniqueness of the formal series $\varepsilon(h)$ is proved in a geometrical way in [6]: we associate to the microlocal situation a natural invariant using a cross-ratio.

- We will prove formula (14). Because $H_{0}$ vanishes and is critical at the point $s$, the sub-principal symbol $H_{1}(s)$ of $\hat{H}$ at $s$ is the same as that of $\hat{W}\left(\hat{y \eta}-h \varepsilon_{0}\right)$ at $O$. The last one is $-W(O) \varepsilon_{0}$. In order to evaluate $W(O)$, we compute the determinant of the Hessian of $H_{0}$ at the critical point which is preserved by $\chi$. We find:

$$
|W(O)|=\left|\operatorname{det}\left(H^{\prime \prime}(s)\right)\right|^{\frac{1}{2}} .
$$

For applications, it may be better not to assume $W(O)>0$. We prefer to assume that the image by $\chi$ of the first quadrant is pointing to the top (see Fig. 8), then $W(O)$ is $>0$ or $<0$ according to the sign of $H_{0}$ in that domain. The sign of $\varepsilon_{0}$ has to be exchanged according to the same rule.

Definition 4. The formal series $\varepsilon(h)=\varepsilon_{0}+O(h)$ is the semi-classical invariant of the saddle point $s$. 
3.2. Microlocal solutions. Using the previous normal form it is easy to describe the microlocal solutions of $\hat{H} u=0$ near the vertex $s$ of $Z_{0}$. We have the following:

Theorem 4. Assuming the same hypothesis as in Theorem 3, the module of microlocal solutions near $s$ is a free module of rank 2. More precisely, taking the choices of Fig. 8 , let us denote by $\mathcal{M}_{j}, j=1,2,3,4$ the free module of dimension 1 of microcal solutions on the edge $e_{j}$ there exists basis $\phi_{j}$ of $\mathcal{M}_{j}$ such that the collection $\left(x_{j} \phi_{j}\right)_{j=1, \cdots, 4}$ extends to a microlocal solution near $s$ if and only if

$$
\left(\begin{array}{l}
x_{3} \\
x_{4}
\end{array}\right)=T(\varepsilon)\left(\begin{array}{l}
x_{1} \\
x_{2}
\end{array}\right),
$$

where $T(\varepsilon)$ is given by

$$
T(\varepsilon)=\mathcal{E}\left(\begin{array}{ll}
1 & i e^{-\varepsilon \pi} \\
i e^{-\varepsilon \pi} & 1
\end{array}\right)
$$

with:

$$
\begin{aligned}
\mathcal{E} & =\frac{1}{\sqrt{2 \pi}} \Gamma\left(\frac{1}{2}+i \varepsilon\right) e^{\varepsilon\left(\frac{\pi}{2}+i \ln h\right)} \\
& =\frac{1}{\sqrt{1+e^{-2 \pi \varepsilon}}} e^{i \arg \Gamma(1 / 2+i \varepsilon)+i \varepsilon \ln (h)} .
\end{aligned}
$$

Proof. Using Eq. (12), it is enough to prove the result for $\hat{H}_{0}=\hat{y \eta}-h \varepsilon(h)$ and to find microlocal solutions $\varphi_{j}$ of $\hat{H}_{0}$ which satisfies the statements of the theorem. The $\varphi_{j}$ 's are small variations of the formulae given in [7], formulae are as follows:

$$
\begin{aligned}
& \varphi_{1}(y)=Y(y)|y|^{-1 / 2} e^{i \varepsilon \ln |y|}, \\
& \varphi_{2}(y)=Y(-y)|y|^{-1 / 2} e^{i \varepsilon \ln |y|}, \\
& \varphi_{3}(y)=\int^{\star} Y(\eta)|\eta|^{-1 / 2} e^{i \frac{y \eta}{h}} e^{-i \varepsilon \ln |\eta|} d \eta, \\
& \varphi_{4}(y)=\int^{\star} Y(-\eta)|\eta|^{-1 / 2} e^{i \frac{y \eta}{h}} e^{-i \varepsilon \ln |\eta|} d \eta,
\end{aligned}
$$

where

$$
\int^{\star}=(2 \pi h)^{-1 / 2} e^{-i \pi / 4} \int
$$

and $Y$ is the Heaviside's function. The $\varphi_{j}$ 's are generators of the microlocal solutions of Eq. (6) on the edges $e_{j}^{\prime}$ (see Fig. 8).

We define then $\phi_{j}=\hat{U} \varphi_{j}$. 


\section{Sheaves on $Z$}

4.1. Linear algebra. Let $Z$ be a connected tetravalent graph, embedded in the plane $X$, which may have some open edges. Let us denote by $e$ the generic edge. We also assume that we have chosen for each vertex some labeling of the four attached edges: $e_{1}, e_{2}, e_{3}, e_{4}$ (for example, by following the convention of Fig. 8). We will always assume that the direct cyclic order is $(1,3,2,4)$.

Definition 5. We define a sheaf $\mathcal{F}=\left(Z, E_{e}, F_{s}\right)$ on $Z$ in the following way: to each edge $e$, we associate a 1-dimensional vector space $E_{e}$ over a field $K$ (or a free modulus over some ring) and, to each vertex s, we associate a generic 2-dimensional subspace $F_{s}$ of the direct sum $\oplus_{j=1}^{4} E_{e_{j}}$. Generic means that $F_{s}$ is not included into a sum of 3 of the $E_{e_{j}}$ and does not contain any lines $E_{e_{j}} \oplus 0$.

We can interpret $F_{s}$ as the graph of a linear mapping $T_{s}: E_{e_{1}} \oplus E_{e_{2}} \rightarrow E_{e_{3}} \oplus E_{e_{4}}$. There exists some basis, which we call standard basis of the $E_{e_{j}}$ such that the matrix of $T$ satisfies $T_{i, j}=1$ for $(i, j)=(1,4),(2,3),(2,4)$ and $T_{1,3}=\rho_{s}$ :

$$
T=\left(\begin{array}{ll}
\rho_{s} & 1 \\
1 & 1
\end{array}\right) .
$$

$\rho_{s}$ is a non-zero scalar which is independent of the chosen basis: $\rho_{s}=\frac{T_{1,3} T_{2,4}}{T_{1,4} T_{2,3}}$. We may interpret it using some cross-ratio as in [6]. Let us define the lines $D_{j} \subset F_{s}$ as the vectors whose projection on $E_{e_{j}}$ vanishes. Then $\rho_{s}$ is the cross-ratio of the four lines $D_{j}$. Standard basis are unique up to some global similarity.

Such a choice of standard basis can be interpreted as a sub-sheaf $\mathcal{F}_{1}$ of $\mathcal{F}$ on $Z$. The germs of $\mathcal{F}_{1}$-sections at each vertex is now of dimension 1 . Let $\gamma$ be a cycle of $Z$. We associate with it a non-zero scalar hol $(\gamma)$ which is the holonomy along $\gamma$ of the sheaf $\mathcal{F}_{1}$ defined using the following prescription: if $\phi_{+}$is a section on the edge $e\left(\phi_{+} \in E_{e} \backslash 0\right)$ which we follow as section of $\mathcal{F}_{1}$ along $\gamma$, we get a new vector $\phi_{-}$in $E_{e}$ and we put:

Definition 6. The holonomy $\operatorname{hol}(\gamma)$ of $\gamma$ is then defined by:

$$
\phi_{-}=\operatorname{hol}(\gamma) \phi_{+}
$$

We then have:

Theorem 5. The $n+N$ non-zero scalars $\rho_{s}$ and $\operatorname{hol}(\gamma)$ define the sheaf $\mathcal{F}=\left(Z, E_{e}, F_{s}\right)$ up to isomorphism. If we call the section of $\mathcal{F}$ the data of one vector of $\oplus E_{e}$ which belongs to $F_{s}$ for each vertex, the dimension of the space of sections $b_{0}(\mathcal{F})$ is only dependent of these invariants. The same statement is true for the $\mathcal{S}$-matrix (up to conjugacy by diagonal matrices) in case where $Z$ admits some open edges.

Proof. By cutting some edges of $Z$, we get a maximal tree $T$ of $Z$. Each oriented cut edge is associated to some unique cycle $\gamma \in H_{1}(Z, \mathbb{Z})$ and that way we get a bijection between the set of cut edges and a basis of cycles of $Z$. The data permit to trivialize the sheaf $\mathcal{F}_{1}$ on $T$ and to rebuild $\mathcal{F}$ on $T$. Holonomies hol $(\gamma)$ can then be read on the cut edges using Definition 6. 


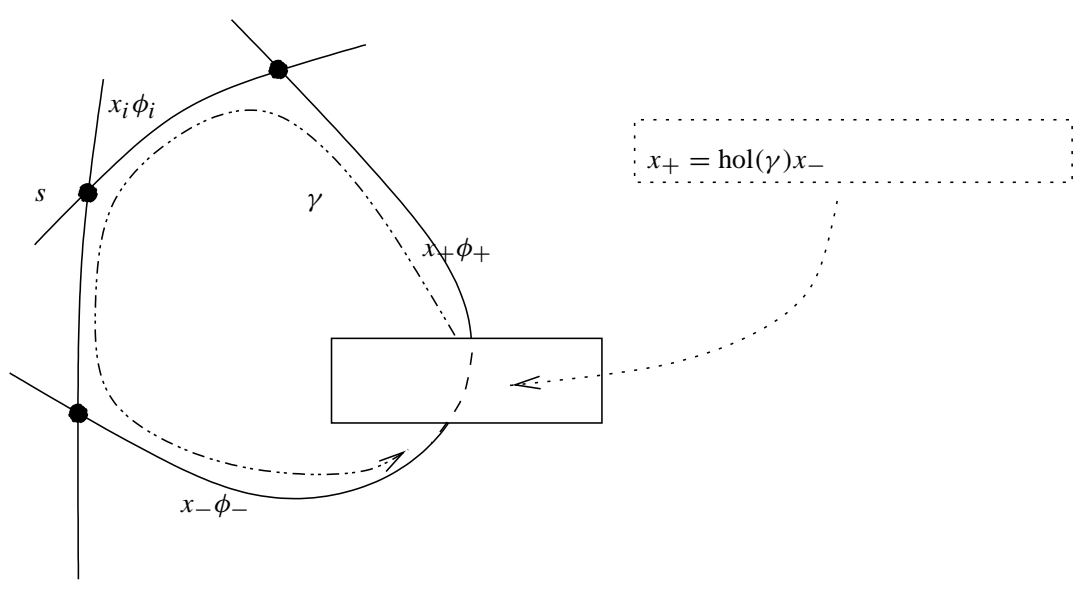

Fig. 9. Recipe for the holonomies

4.2. The semi-classical case. Moduli $F_{S}$ are given by microlocal solutions near the saddle point $s$. The number $\rho$ is computed in terms of the series $\varepsilon(h)$ associated to the saddle point by Theorem 3. More precisely, we get

$$
\rho_{s}=-e^{2 \pi \varepsilon} \text {. }
$$

Of course, we may have used another normal form in order to define the sub-sheaf $\mathcal{F}_{1}$.

We then take the normal form given in Eq. (17) for the definition of a standard basis.

As $\varepsilon \rightarrow \pm \infty$, Stirling's formula allows to show the following asymptotics;

$$
T(\varepsilon)=e^{i \varepsilon \ln |h \varepsilon|}\left(\begin{array}{ll}
1 & 0 \\
0 & 1
\end{array}\right)\left(1+O\left(\frac{1}{\varepsilon}\right)\right), \varepsilon \rightarrow+\infty,
$$

and

$$
T(\varepsilon)=e^{i \varepsilon \ln |h \varepsilon|}\left(\begin{array}{ll}
0 & i \\
i & 0
\end{array}\right)\left(1+O\left(\frac{1}{\varepsilon}\right)\right), \varepsilon \rightarrow-\infty .
$$

These asymptotics describe the transition between the singular and the smooth case. They allow in particular to check the validity of the quantization rules!

Problem. How should we write the condition $b_{0}(\mathcal{F}) \neq 0$ in terms of these invariants? How should we compute the scattering matrix?

Here is a possible way to derive the quantization rules. We first cut $b_{1}$ edges of $Z$ in order to get a maximal tree $T$. We recall that once chosen $T$ there is a canonical bijection between cut edges and a basis of $H_{1}(Z, \mathbb{Z})$ : to any cut edge is associated the unique nontrivial cycle of $Z$ which is contained in $T$ except for this edge. We choose a non-zero section $\phi$ of $\mathcal{F}_{1}$ on $T$. We then try to get a solution such that $\psi_{\mid e}=x_{e} \phi_{e}, e \in E(T)$. The $\left(x_{e}\right)$ 's should satisfy

- at each vertex, we have

$$
\left(\begin{array}{l}
x_{e_{3}} \\
x_{e_{4}}
\end{array}\right)=T(\varepsilon)\left(\begin{array}{l}
x_{e_{1}} \\
x_{e_{2}}
\end{array}\right)
$$


- $x_{e_{+}}=(\operatorname{hol}(\gamma)) x_{e_{-}}$for any pair of edges $\left(e_{+}, e_{-}\right)$of $T$ which comes by cutting the edge of $Z$ associated with the cycle $\gamma$.

Putting these last equations in the first system produces a system of $v=2 n$ homogeneous linear equations with $v$ unknown. The quantization rule can then be written as the vanishing of a $v \times v$ determinant where $v=2 n$.

The self-adjoint case. If each space $E_{e}$ is equipped with an Hermitian metric and if the $T_{S}$ are unitary maps, it is possible to choose as a normal form for $T_{S}$ a unitary matrix and the moduli of the holonomies hol $(\gamma)$ are 1: they are just phase shifts. It is easy to check that the condition $b_{0} \neq 0$ can be rewritten as follows: " 1 is an eigenvalue of a unitary matrix". We can take for example the $\mathcal{S}$ matrix associated to a maximal tree of $Z$.

Let us assume that we are looking at eigenvalues of a Schrödinger operator $K$ (cf. Sect. 1.3). The equation $b_{0} \neq 0$ is equivalent to 1 is an eigenvalue of a unitary matrix which depends of the spectral parameter $\lambda$. This equation with complex entries depends on a real parameter. In fact this equation is equivalent to a real one, the singular Bohr-Sommerfeld rule: if we put $K=\{A \in U(n) \mid 1 \in \sigma(A)\}, K$ is a codimension 1 submanifold of $U(n)$.

\section{Computation of the Holonomies up to $O(h)$}

We should first compute $\rho_{s}$ and the holonomies hol $(\gamma)$ as in $\S 4$. We have already seen that $\rho_{s}=-e^{2 \pi \varepsilon}$. We are left with the computation of holonomies, which is the main statement of this paper:

Theorem 6. Assuming the choice of the normal form given by Eq. (17) and the conventions of Fig. 8, the holonomies hol $(\gamma)$ are given by:

$$
\operatorname{hol}(\gamma)=e^{\frac{i}{h} A_{\gamma}-i I_{\gamma}+i \frac{\pi}{2} m(\gamma)}(1+O(h)),
$$

where $A_{\gamma}, I_{\gamma}$ and $m(\gamma)$ are defined in Sect. 2.

\section{Justification}

Let $\varphi_{j}(y), j=1,2,3,4$ be the functions defined by Eq. (20). Then let $f$ be a microlocal solution in some neighborhood of $O$ of the equation

$$
(\hat{y \eta}-h \varepsilon) f=O\left(h^{\infty}\right),
$$

$f$ is microlocally outside of 0 equal to $y_{j} \varphi_{j}$ on $e_{j}^{\prime}$. We have then:

$$
\left(\begin{array}{l}
y_{3} \\
y_{4}
\end{array}\right)=T(\varepsilon)\left(\begin{array}{l}
y_{1} \\
y_{2}
\end{array}\right) \text {. }
$$

Let us put (assuming we are in the generic case and restricting to the edges $e_{1}$ and $\left.e_{4}\right)$ :

$$
\chi^{\prime}(O)=\left(\begin{array}{ll}
a & b \\
c & d
\end{array}\right) \text { with } a>0, b<0,
$$

where $\chi$ is the canonical map of Theorem 3 (see Fig. 8). 
We may assume that the singular point $s$ is the origin $O$. Let $S(x, y)=S_{0}(x, y)+$ $O\left((|x|+|y|)^{3}\right)$ be the generating function of $\chi$ defined by:

$$
\chi\left(y,-\frac{\partial S}{\partial y}\right)=\left(x, \frac{\partial S}{\partial x}\right)
$$

and $S(O)=0$. We get:

$$
S_{0}(x, y)=\frac{1}{2 b}\left(d x^{2}-2 x y+a y^{2}\right) .
$$

The FIO $\hat{U}$ is then given by:

$$
\hat{U} f(x)=\int^{\star} e^{\frac{i}{h} S(x, y)} C_{h}(x, y) f(y) d y,
$$

where $C_{h}(x, y) \sim \sum_{j=0}^{\infty} C_{j}(x, y) h^{j}$ and $C_{0}(O)=|b|^{-\frac{1}{2}}$.

Let us define $\phi_{j}=\hat{U} \varphi_{j}$. By Eq. (12), the $\phi_{j}$ 's are microlocal solutions of $\hat{H}$ on the $e_{j}$ 's.

We compute the WKB form of $\phi_{1}$ (resp. $\phi_{4}$ ) for $x_{1}>0$ (resp. $x_{4}>0$ ), but close to 0 , in order to compute their phase shift. We apply the stationary phase method to the integral (where $x_{1}>0$ ):

$$
\phi_{1}\left(x_{1}\right)=\int^{\star} e^{\frac{i}{h} S\left(x_{1}, y\right)} C_{h}\left(x_{1}, y\right) \varphi_{1}(y) g(y) d y,
$$

where $g(y) \in C_{0}^{\infty}(\mathbb{R} \backslash 0)$ is 1 near $y_{0}$ and such that $\chi\left(y_{0}, 0\right)=\left(x_{1}, \xi_{1}\right)=z_{1}$. We get:

$$
\phi_{1}\left(x_{1}\right)=-i e^{\frac{i}{h} S_{1}\left(x_{1}\right)} e^{i \varepsilon \ln \left|\frac{x_{1}}{a}\right|}\left|\frac{1}{x_{1}}\right|^{\frac{1}{2}}\left(A_{0}\left(x_{1}\right)+O(h)\right),
$$

where $A_{0}(x)=1+O(x)$ and $S_{1}\left(x_{1}\right)=\int_{0}^{z_{1}} \xi d x$ is the generating function of the arc $\chi(\mathbb{R} \times 0)$ which vanishes at $x=0$. In the same way, for $x_{4}>0$,

$$
\phi_{4}\left(x_{4}\right)=-i e^{\frac{i}{h} S_{4}\left(x_{4}\right)} e^{-i \varepsilon \ln \left|\frac{x_{4}}{b}\right|}\left|\frac{1}{x_{4}}\right|^{\frac{1}{2}}\left(B_{0}\left(x_{4}\right)+O(h)\right),
$$

where $B_{0}(x)=1+O(x)$ and $S_{4}\left(x_{4}\right)=\int_{0}^{z_{4}} \xi d x$ is the generating function of the arc $\chi(0 \times \mathbb{R})$ which vanishes at $x=0$. We now already from 1.4 .1 that $A_{0}(x) /|x|^{\frac{1}{2}}|d x|^{\frac{1}{2}}=$ $a_{0}(x)|d x|^{\frac{1}{2}}$ lifted to $Z$ is invariant by the flow. Knowing that $\lim _{x_{1} \rightarrow 0} A_{0}\left(x_{1}\right)=1$ implies that this half-density is the canonical one. The same is true for $\phi_{4}$ with $b_{0}(x)|d x|^{\frac{1}{2}}$.

Let us evaluate $\phi_{1}\left(x_{A}\right)$ (resp. $\phi_{4}\left(x_{B}\right)$ ) starting from their values at $x_{1}$ (resp. $x_{4}$ ) in order to calculate their phase shift, using the usual WKB formulae.

Putting $z_{j}=\left(x_{j}, S_{j}^{\prime}\left(x_{j}\right)\right), j=1,4$, we get:

$$
\phi_{1}\left(x_{A}\right)=-i a_{0}\left(x_{A}\right) e^{\frac{i}{h} \int_{s}^{A} \xi d x+i \varepsilon \ln \frac{x_{1}}{a}-i \int_{z_{1}}^{A} H_{1} d t}(1+O(h)),
$$

where the integrals are computed on $Z$, in the same way:

$$
\phi_{4}\left(x_{B}\right)=-i b_{0}\left(x_{B}\right) e^{\frac{i}{h} \int_{s}^{B} \xi d x-i \varepsilon \ln \frac{x_{4}}{|b|}-i \int_{z_{4}}^{B} H_{1} d t}(1+O(h)) .
$$




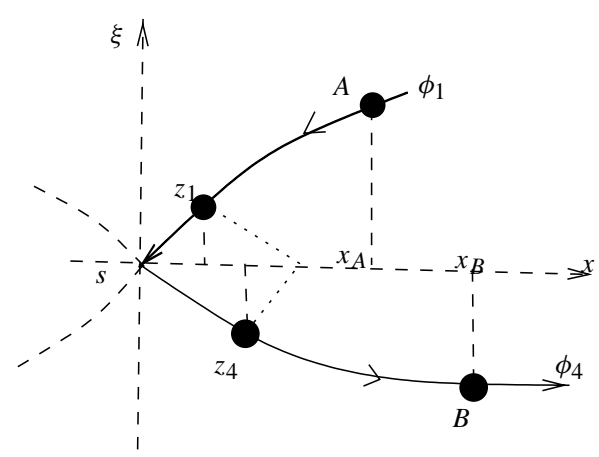

Fig. 10. Computation of the holonomy

Using the preceding computations, we can find the phase shift

$$
\begin{gathered}
\frac{\phi_{4}\left(x_{B}\right)}{b_{0}\left(x_{B}\right)}=e^{i \Omega} \frac{\phi_{1}\left(x_{A}\right)}{a_{0}\left(x_{A}\right)}, \\
\Omega=\frac{1}{h} \int_{A}^{B} \xi d x+\int_{z_{1}}^{A} H_{1} d t-\int_{z_{4}}^{B} H_{1} d t+\varepsilon \ln \left|\frac{x_{4} x_{1}}{a b}\right| .
\end{gathered}
$$

Going to the limit where $z_{1}, z_{4} \rightarrow s$ and using $\left|\omega\left(s z_{1}, s z_{4}\right)\right| \sim \frac{x_{1} x_{4}}{a|b|}$ and the definition 2.2, we get:

$$
\Omega=\frac{1}{h} \int_{A}^{B} \xi d x-\mathrm{vp} \int_{A}^{B} H_{1} d t+O(h)
$$

\section{Summary of Quantization Rules}

Summarizing, we start with a semi-classical Hamiltonian whose principal symbol $H_{0}$ is real and whose sub-principal symbol is denoted by $H_{1}$. We first look at the characteristic manifold $Z=H_{0}^{-1}(0)$, and calculate the invariants $\varepsilon$ of the saddle points. They are formal series in $h$ whose first term $\varepsilon_{0}$ is given by the formula (14). We then choose some maximal tree $T$ of $Z$ and some orientation of the cut edges. It is then possible to calculate the holonomies of the corresponding cycles using formula (29). We then describe any microlocal solution in terms of the trivialisation of $\mathcal{F}_{1}$ in the following way:

$$
u=\oplus_{e} x_{e} u_{e},
$$

where the sum is on the edges of $T$. The conditions on the $x_{e}$ 's are then given by the matrices $T_{\varepsilon}$ associated to each saddle point and the holonomies $x_{e_{+}}=\operatorname{hol}(\gamma) x_{e_{-}}$if the cycle $\gamma$ corresponds to the cut edge $e=e_{+} \cup e_{-}$following $e_{-}$and then $e_{+}$. The relative error on these conditions is of order $O\left(h^{\infty}\right)$; it is of order $O(h)$ when we approximate the holonomies using Eq. (29); in that case, it is also possible to replace $\varepsilon$ by $\varepsilon_{0}$ without changing the relative error. 


\section{The Double Well Potential}

8.1. Generic study of the double well potential. In this section, we will apply the previous theory to the situation of [7] and [8] where $X=T^{\star} \mathbb{R}$ and $V: \mathbb{R} \rightarrow \mathbb{R}$ is a function with a double well. Without loss of generality, we may suppose that:

$$
V(0)=0, V^{\prime}(0)=0, \mathcal{V}=V^{\prime \prime}(0)<0,
$$

e.g. $V(x)=x^{2}\left((x-b)^{2}-a^{2}\right)$ with $a>b>0$.

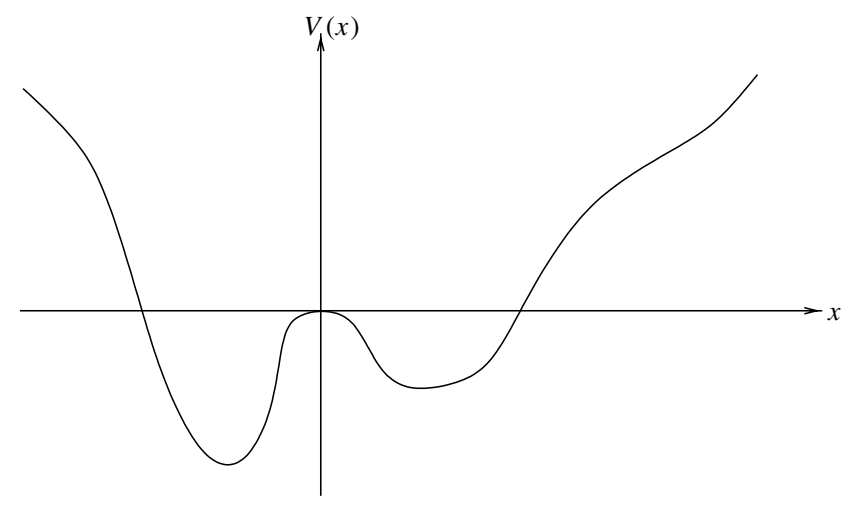

Fig. 11. A double-well potential

Let

$$
\hat{H}=-\frac{h^{2}}{2} \frac{d^{2}}{d x^{2}}+V(x)-\lambda h
$$

be the corresponding Schrödinger operator. A normalized solution of $\hat{H} u=O\left(h^{\infty}\right)$ exists if and only if $\lambda h$ is an eigenvalue of $-\frac{h^{2}}{2} \frac{d^{2}}{d x^{2}}+V(x)$ up to a relative error of order $O\left(h^{\infty}\right)$ (relative to the closest eigenvalue).

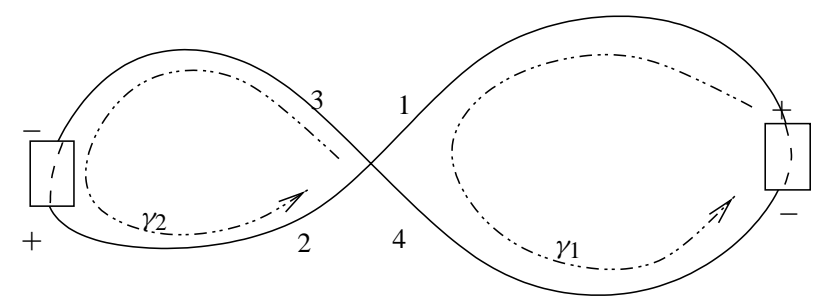

Fig. 12. The double well potential in the phase space

We have:

$$
\operatorname{det}\left(H^{\prime \prime}{ }_{0}\right)=\mathcal{V}, \quad \varepsilon_{0}=\frac{\lambda}{\sqrt{-\mathcal{V}}}
$$


Let us denote by $h_{j}=e^{i \theta_{j}}$ the holonomies of the loops $\gamma_{j}$. The holonomy equations read:

$$
x_{1}=h_{1} x_{4}, x_{2}=h_{2} x_{3},
$$

and the critical point relations are:

$$
\left(\begin{array}{l}
x_{3} \\
x_{4}
\end{array}\right)=T(\varepsilon)\left(\begin{array}{l}
x_{1} \\
x_{2}
\end{array}\right) .
$$

We get the following quantization relation:

$$
\operatorname{det}\left(\left(\begin{array}{ll}
0 & h_{2}^{-1} \\
h_{1}^{-1} & 0
\end{array}\right)-T(\varepsilon)\right)=0,
$$

or equivalently " 1 is an eigenvalue of the matrix":

$$
T(\varepsilon)\left(\begin{array}{ll}
0 & h_{1} \\
h_{2} & 0
\end{array}\right), \quad T(\varepsilon)=\mathcal{E}\left(\begin{array}{ll}
1 & i e^{-\varepsilon \pi} \\
i e^{-\varepsilon \pi} & 1
\end{array}\right) .
$$

This matrix is unitary, hence we may apply [8, Appendix] and rewrite the quantization rule as a real equation, like [8, Proposition 3]:

Proposition 1. The equation $\hat{H} u=O\left(h^{\infty}\right)$ has a normalized eigenfunction if and only if $\lambda$ satisfies the following condition:

$$
\frac{1}{\sqrt{1+e^{2 \pi \varepsilon}}} \cos \left(\frac{\theta_{1}-\theta_{2}}{2}\right)=\cos \left(\frac{\theta_{1}+\theta_{2}}{2}+\frac{\pi}{2}+\varepsilon \ln h+\arg \Gamma\left(\frac{1}{2}+i \varepsilon\right)\right),
$$

where $\varepsilon=\varepsilon_{0}+O(h)(c f .(11)$ and (34)) and:

$$
e^{i \theta_{1}}=h_{1}, \quad e^{i \theta_{2}}=h_{2}
$$

with

$$
\theta_{j}=\frac{1}{h} \int_{\gamma_{j}} \xi d x-\lambda v \mathrm{vp} \int_{\gamma_{j}} d t+\frac{\pi}{2}+O(h) .
$$

We want to calculate more explicitly (38) up to a relative error of order $O(h)$. Hence we replace $\varepsilon$ by $\varepsilon_{0}$, and calculate $\theta_{1}$ and $\theta_{2}$ using (29). We observe that the Maslov indices are +1 and that the $A_{\gamma_{i}}$ values are opposite to the area inside the cycles $\gamma_{i}$ (since $d(\xi d x)=-d x \wedge d \xi)$. It remains to do the calculation of the regularized integrals $I_{\gamma_{i}}$.

Note that along a trajectory of the Hamiltonian $H=\xi^{2} / 2+V(x)$ one has:

$$
\frac{d x}{d t}=\xi_{ \pm}= \pm \sqrt{-2 V(x)} .
$$

Let $\gamma$ denote one of the two cycles, let $A$ be the intersection of $\gamma$ with the $x$ axis. Let $x_{A}$ be the $x$-coordinate of $A$ (e.g. for $V(x)=x^{2}\left((x-b)^{2}-a^{2}\right), x_{A}=b \pm a$ ). From Definition (8), we get:

$$
\begin{aligned}
I_{\gamma} & =\lim _{\tilde{a} \tilde{b} \rightarrow s_{j}}\left(\int_{A}^{\tilde{a}} H_{1} d t+\int_{\tilde{b}}^{A} H_{1} d t+a(0) \ln \int_{R_{\tilde{a}, \tilde{b}}} \omega\right) \\
& =\lambda \lim _{x \rightarrow 0}\left(2 \int_{x_{A}}^{x} \frac{-d X}{\sqrt{-2 V(X)}}+\frac{1}{\sqrt{-\mathcal{V}}} \ln |2 x \sqrt{-2 V(x)}|\right) .
\end{aligned}
$$


8.2. Non-singular limit. In this section, we show that the limit of the singular BohrSommerfeld quantization rules as $\varepsilon \rightarrow \pm \infty$ are the usual quantization rules.

We apply the Stirling formula:

$$
\arg \Gamma\left(\frac{1}{2}+i \varepsilon\right)=\varepsilon \ln |\varepsilon|-\varepsilon+o(1)
$$

and evaluate the area inside the curve $\frac{\xi^{2}}{2}+V(x)=\tau$ as $\tau$ tends to 0 . From Eq. (10) (Sect. 2.2), we get:

$$
A(\tau)=A(0)+2 \frac{\tau}{\sqrt{|\mathcal{V}|}}\left(1-\ln \frac{|\tau|}{\sqrt{|\mathcal{V}|}}\right)-\left(I_{\gamma_{1}}+I_{\gamma_{2}}\right) \tau+O\left(\tau^{2} \ln |\tau|\right),
$$

where $H_{1}=1$ in the definition of the $I_{\gamma}$ integrals.

Here $\tau=\lambda h$ and we must change the sign of $A(\tau)$ since the cycles are oriented using the trigonometric convention:

$$
\begin{aligned}
\int_{H=\lambda h} \xi d x & =\int_{H=0} \xi d x+2 \frac{\lambda h}{\sqrt{|\mathcal{V}|}}\left(\ln \frac{|\lambda h|}{\sqrt{|\mathcal{V}|}}-1\right)+\left(I_{\gamma_{1}}+I_{\gamma_{2}}\right) \lambda h+O\left(\lambda^{2} h^{2} \ln h\right) \\
& =\int_{H=0} \xi d x+2 \varepsilon_{0} h\left(\ln \left|h \varepsilon_{0}\right|-1\right)-h\left(I_{\gamma_{1}}+I_{\gamma_{2}}\right) H_{1}+O\left(\lambda^{2} h^{2} \ln h\right),
\end{aligned}
$$

where $H_{1}=-\lambda$.

If $\lambda$ tends to $+\infty$, Eq. (38) is satisfied if the argument of the cosine of the right-hand side is $\pi / 2$ modulo $\pi$, hence:

$$
\frac{\theta_{1}+\theta_{2}}{2}+\varepsilon \ln h-\varepsilon+\varepsilon \ln \varepsilon+o_{\varepsilon}(1)=k \pi
$$

On the other hand:

$$
\begin{aligned}
\frac{\theta_{1}+\theta_{2}}{2} & =\frac{\pi}{2}+\frac{1}{2 h} \int_{H=0} \xi d x-\frac{1}{2}\left(I_{\gamma_{1}}+I_{\gamma_{2}}\right)\left(H_{1}\right) \\
& =\frac{\pi}{2}+\frac{1}{2 h} \int_{H=\lambda h} \xi d x-\varepsilon_{0}\left(\ln \left|h \varepsilon_{0}\right|-1\right)+O\left(h \ln h \varepsilon_{0}^{2}\right)
\end{aligned}
$$

therefore (39) becomes:

$$
\frac{1}{2 h} A(h \varepsilon)+\frac{\pi}{2}+O\left(h \ln h \varepsilon^{2}\right)+o_{\varepsilon \rightarrow+\infty}(1)=k \pi
$$

which is the usual Bohr-Sommerfeld quantization rule. We leave the same verification as $\lambda$ tends to $-\infty$ as an exercise for the reader.

8.3. Analytic calculations for polynomial potentials of degree 4. In this section we examine further the example $V(x)=x^{2}\left((x-b)^{2}-a^{2}\right)$. 
8.3.1. Results. Using our preferred CAS (computer algebra system), we determine analytically the $A_{\gamma_{i}}$ 's:

$$
\begin{aligned}
& A_{\gamma_{d}}=\sqrt{2}\left(-\frac{2{\sqrt{a^{2}-b^{2}}}^{3}}{3}-\sqrt{a^{2}-b^{2}} b^{2}-b a^{2} \arcsin \left(\frac{b}{a}\right)-\frac{a^{2} b \pi}{2}\right), \\
& A_{\gamma_{g}}=\sqrt{2}\left(-\frac{2{\sqrt{a^{2}-b^{2}}}^{3}}{3}-\sqrt{a^{2}-b^{2}} b^{2}-b a^{2} \arcsin \left(\frac{b}{a}\right)+\frac{a^{2} b \pi}{2}\right)
\end{aligned}
$$

and $I_{\gamma}$ 's:

$$
I_{\gamma_{d}}=\varepsilon_{0}\left(\frac{7}{2} \ln 2\left(a^{2}-b^{2}\right)-2 \ln a\right)=\varepsilon_{0}\left(\frac{7}{2} \ln \left(2\left(a^{2}-b^{2}\right)\right)-2 \ln a\right) .
$$

We observe that $I_{\gamma_{g}}=I_{\gamma_{d}}$, this will be explained in Sect. 8.3.2.

Hence (29) reads, up to a relative error of order $O(h)$ :

$$
\begin{aligned}
\theta_{1}-\theta_{2}= & \sqrt{2} \frac{a^{2} b \pi}{h}, \\
\theta_{1}+\theta_{2}= & \pi-2 \varepsilon_{0}\left(\frac{7}{2} \ln \left(2\left(a^{2}-b^{2}\right)\right)-2 \ln a\right)+ \\
& +\sqrt{2} \frac{2}{h}\left(-\frac{2{\sqrt{a^{2}-b^{2}}}^{3}}{3}-\sqrt{a^{2}-b^{2}} b^{2}-b a^{2} \arcsin \left(\frac{b}{a}\right)\right),
\end{aligned}
$$

then we apply (38) and we get:

Proposition 2. The solutions $\varepsilon_{0}$ of:

$$
\begin{aligned}
\frac{1}{\sqrt{1+e^{2 \pi \varepsilon_{0}}}} \cos \left(\frac{a^{2} b \pi}{2 h}\right)= & \cos \left(\pi-2 \varepsilon_{0}\left(\frac{7}{2} \ln \left(2\left(a^{2}-b^{2}\right)\right)-2 \ln a\right)+\right. \\
& +\frac{\sqrt{2}}{h}\left[-\frac{2{\sqrt{a^{2}-b^{2}}}^{3}}{3}-\sqrt{a^{2}-b^{2}} b^{2}-b a^{2} \arcsin \left(\frac{b}{a}\right)\right]+ \\
& \left.+\varepsilon_{0} \ln h+\arg \Gamma\left(\frac{1}{2}+i \varepsilon_{0}\right)\right)
\end{aligned}
$$

correspond to the eigenvalues $\lambda h=\sqrt{2\left(a^{2}-b^{2}\right)} \varepsilon_{0} h$ of the Schrödinger operator

$$
H=-\frac{h^{2}}{2} \frac{d^{2}}{d x^{2}}+V(x)=x^{2}\left((x-b)^{2}-a^{2}\right)
$$

up to a relative error of order $O(h)$ (relative means relative to the closest eigenvalue).

If we want to get the numerical values of the eigenvalues, we are left to solve (41). In Appendix A, we will compare the corresponding eigenvalues $\lambda h$ to the eigenvalue that we calculate using purely numerical techniques (Runge-Kutta's method here) to show the accuracy of the singular Bohr-Sommerfeld quantization rules. The semi-classical method is very useful for small values of $h$ because the computation time is essentially independent of $h$, as it grow linearly for the Runge-Kutta method since we have to choose a step of size $\varepsilon h$ (therefore we must make $1 /(\varepsilon h)$ steps). 
8.3.2. Equality $I_{\gamma_{d}}=I_{\gamma_{g}}$. The equality $I_{\gamma_{g}}=I_{\gamma_{d}}$ for polynomial potentials of degree 4 can be shown directly. From (10), we have to show that $A_{+}(E)-A_{-}(E)=$ constant, where $A_{ \pm}(E), E<0$, stand for the areas of the two components of $\left\{(x, \xi) / \xi^{2}+V(x) \leq\right.$ $E\}$. Let $\gamma_{ \pm}$denote the border of these two domains, one has:

$$
\frac{d A_{ \pm}}{d E}=\int_{\gamma_{ \pm}} \frac{d x}{\xi} .
$$

The 1-form $\frac{d x}{\xi}$ is holomorphic on the smooth holomorphic projective compactification of the curve $\xi^{2}+V(x)=E$. This curve is a torus if $V$ is a polynomial of degree 4 , hence the curves $\gamma_{+}$and $\gamma_{-}$are homotopic (for they are non degenerate and they don't intersect), which completes the proof of (42).

\section{The Triple Well Potential}

Same context as in Sect. 8, but $V$ is a potential with three wells so that the corresponding saddle points are at the same height.

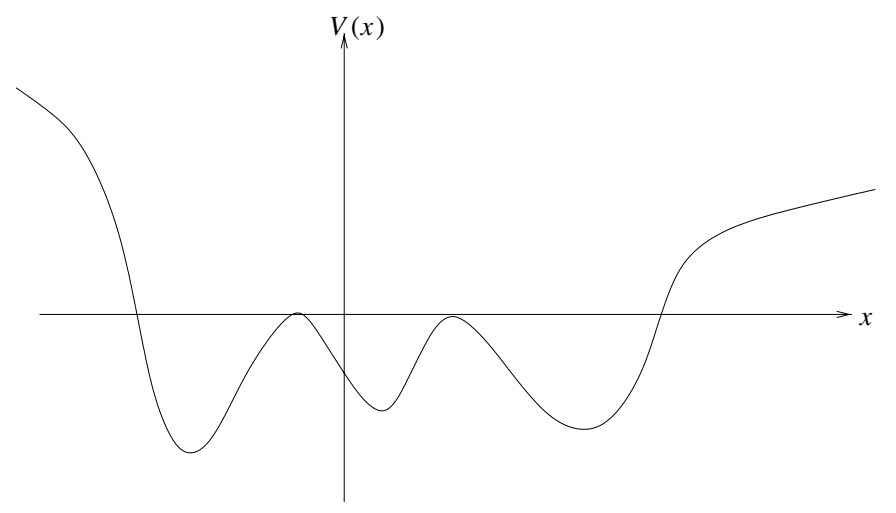

Fig. 13. A triple well potential

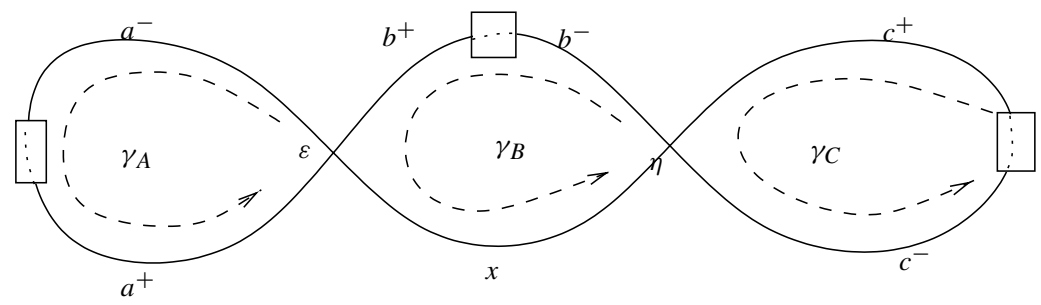

Fig. 14. The triple well potential in the phase space

We construct a maximal tree (see Fig. 14) with 7 edges denoted by $a^{ \pm}, b^{ \pm}, c^{ \pm}$and $x$. The holonomy equations corresponding to the three cycles $\gamma_{A}, \gamma_{B}$ and $\gamma_{C}$ are:

$$
a^{+}=\alpha a^{-}, \quad b^{+}=\beta b^{-}, \quad c^{+}=\gamma c^{-} .
$$


Let $\varepsilon$ and $\eta$ denote the semi-classical invariants of the two vertices, the following relations hold:

$$
\left(\begin{array}{c}
a^{-} \\
x
\end{array}\right)=T(\varepsilon)\left(\begin{array}{l}
b^{+} \\
a^{+}
\end{array}\right), \quad\left(\begin{array}{l}
b^{-} \\
c^{-}
\end{array}\right)=T(\eta)\left(\begin{array}{c}
c^{+} \\
x
\end{array}\right) .
$$

Hence:

$$
\left(\begin{array}{cc}
T(\varepsilon) & 0 \\
0 & T(\eta)
\end{array}\right)\left(\begin{array}{c}
b^{+} \\
a^{+} \\
c^{+} \\
x
\end{array}\right)-\left(\begin{array}{c}
a^{-} \\
x \\
b^{-} \\
c^{-}
\end{array}\right)=0
$$

Since:

$$
\left(\begin{array}{c}
a^{-} \\
x \\
b^{-} \\
c^{-}
\end{array}\right)=\left(\begin{array}{cccc}
0 & \alpha^{-1} & 0 & 0 \\
0 & 0 & 0 & 1 \\
\beta^{-1} & 0 & 0 & 0 \\
0 & 0 & \gamma^{-1} & 0
\end{array}\right)\left(\begin{array}{c}
b^{+} \\
a^{+} \\
c^{+} \\
x
\end{array}\right)
$$

the system has a non-trivial solution if and only if:

$$
\operatorname{det}\left(\left(\begin{array}{cc}
T(\varepsilon) & 0 \\
0 & T(\eta)
\end{array}\right)-\left(\begin{array}{cccc}
0 & \alpha^{-1} & 0 & 0 \\
0 & 0 & 0 & 1 \\
\beta^{-1} & 0 & 0 & 0 \\
0 & 0 & \gamma^{-1} & 0
\end{array}\right)\right)=0
$$

Let $\mathcal{E}$ and $\mathcal{N}$ denote the common factors of the coefficients of the matrices $T(\varepsilon)$ and $T(\eta)$ defined by (18) for $\mathcal{E}$ and a similar formula for $\mathcal{N}$. Dividing the first two lines of the determinant by $\mathcal{E}$ and the last two lines by $\mathcal{N}$, we get:

$$
\left|\begin{array}{llcc}
1 & i e^{-\varepsilon \pi}-\frac{1}{\alpha \mathcal{E}} & 0 & 0 \\
i e^{-\varepsilon \pi} & 1 & 0 & \frac{-1}{\mathcal{E}} \\
\frac{-1}{\beta \mathcal{N}} & 0 & 1 & i e^{-\eta \pi} \\
0 & 0 & i e^{-\eta \pi}-\frac{1}{\gamma \mathcal{N}} 1
\end{array}\right|=0 .
$$

Now we expand the determinant with respect to the first column:

$$
\begin{array}{r}
\left(1-i e^{-\eta \pi}\left(i e^{-\eta \pi}-\frac{1}{\gamma \mathcal{N}}\right)\right)\left(1-i e^{-\varepsilon \pi}\left(i e^{-\varepsilon \pi}-\frac{1}{\alpha \mathcal{E}}\right)\right)+ \\
\frac{1}{\beta \mathcal{N}}\left(i e^{-\varepsilon \pi}-\frac{1}{\alpha \mathcal{E}}\right) \frac{-1}{\mathcal{E}}\left(i e^{-\eta \pi}-\frac{1}{\gamma \mathcal{N}}\right)=0 .
\end{array}
$$

Defining:

$$
A=e^{-\varepsilon \pi}+i \frac{1}{\alpha \mathcal{E}}, \quad C=e^{-\eta \pi}+i \frac{1}{\gamma \mathcal{N}}
$$

we rewrite the previous equation as:

$$
\left(1+e^{-\eta \pi} C\right)\left(1+e^{-\varepsilon \pi} A\right)=-\frac{A C}{\beta \mathcal{N} \mathcal{E}} .
$$


We want to show that this complex equation reduces to a real equation for real values of $E$. For this we will prove that the modulus of both sides are equal. Let us introduce the following notations:

$$
\tilde{\alpha}=-\arg \alpha-\arg \mathcal{E}, \quad \tilde{\gamma}=-\arg \gamma-\arg \mathcal{N} .
$$

Using this notation we may rewrite:

$$
A=e^{-\varepsilon \pi}+\sqrt{1+e^{-2 \varepsilon \pi}} e^{i \tilde{\alpha}}, \quad C=e^{-\eta \pi}+\sqrt{1+e^{-2 \eta \pi}} e^{i \tilde{\gamma}},
$$

since $|\mathcal{E}|=1 / \sqrt{1+e^{-2 \varepsilon \pi}}$. Hence:

$$
\begin{aligned}
1+e^{-\varepsilon \pi} A & =1+e^{-2 \varepsilon \pi}+e^{-\pi \varepsilon} \sqrt{1+e^{-2 \pi \varepsilon}} e^{i \tilde{\alpha}} \\
& =\sqrt{1+e^{-2 \varepsilon \pi}} e^{i \tilde{\alpha}} \bar{A}
\end{aligned}
$$

and Eq. (44) becomes:

$$
e^{i \tilde{\alpha}+i \tilde{\gamma}} \sqrt{1+e^{-2 \varepsilon \pi}} \sqrt{1+e^{-2 \eta \pi}} \overline{A C}=-\frac{A C}{\beta \mathcal{E N}} .
$$

It is now clear that both sides have the same modulus, therefore this equation reduces to:

$$
\tilde{\gamma}+\tilde{\alpha}=\pi-\arg \beta-\arg \mathcal{E}-\arg \mathcal{N}+2 \arg A+2 \arg C \quad(2 \pi) .
$$

Eventually we get:

Proposition 3. The eigenvalues of the triple well potential Schrödinger operator correspond to the solutions $\lambda$ of:

$$
\arg (\beta)-\arg (\alpha)-\arg (\gamma)=\pi+2 \arg A+2 \arg C \quad(2 \pi)
$$

up to a relative error of order $O\left(h^{\infty}\right)$. Here $\alpha, \beta$ and $\gamma$ denote the holonomies of the 3 cycles and A, $C$ as defined in (43), (18).

Exercise. Let $a>0, b>0$ and define:

$$
V(x)=a\left(x^{2}-1\right)^{3}-b\left(x^{2}-1\right)^{2}=\left(x^{2}-1\right)^{2}\left(a x^{2}-a-b\right) .
$$

Calculate the singular Bohr-Sommerfeld quantization rules and compare with purely numerical techniques, as for the quadratic potential of Sect. 8.3.

Hints:

We have:

$$
V^{\prime}(x)=2 x\left(3 a\left(x^{2}-1\right)^{2}-2 b\left(x^{2}-1\right)\right)=2 x\left(x^{2}-1\right)\left(3 a x^{2}-3 a-2 b\right),
$$

hence this potential has five critical points: $0, \pm 1, \pm \sqrt{1+2 b /(3 a)}$. The corresponding critical values are: $-a-b<0,0,-4 b^{3} /\left(27 a^{2}\right)<0$ and the corresponding Hessians are: $6 a+4 b>0,-8 b<0$ and $24 b+16 b^{2} /(3 a)>0$. Hence $V$ has two local maxima at \pm 1 with $V( \pm 1)=0$. It is possible to calculate analytically the action integrals since for $x \in[-1,1]$ :

$$
W(x)=\sqrt{-V(x)}=\left(1-x^{2}\right) \sqrt{b+a-a x^{2}}
$$

and $1 / W$ both admit an explicit anti-derivative. 


\section{Hill's Equation}

In this section we are concerned with the Schrödinger operator

$$
\hat{H}=-\frac{h^{2}}{2} \frac{d^{2}}{d x^{2}}+V(x),
$$

where $V: \mathbb{R} \rightarrow \mathbb{R}$ is a $C^{\infty}$ periodic potential of period $T>0$ such that $\sup _{x \in \mathbb{R}} V(x)=$ 0 .

10.1. Hill's Equation: Spectrum and discriminant. Let us recall some well known facts about the spectrum of the Hill equation and its discriminant.

The operator $\hat{H}$ is essentially self-adjoint and has a continuous spectrum made of the union of intervals called bands separated by gaps.

In order to get quantitative information on these bands, let us introduce the discriminant function $\Delta(E)$. Let $P_{E}: \mathbb{C}^{2} \rightarrow \mathbb{C}^{2}$ be the linear map defined by $P_{E}\left(u(0), u^{\prime}(0)\right)=$ $\left(u(T), u^{\prime}(T)\right)$ where $u$ is a solution of $(\hat{H}-E) u=0$. It is easy to prove that $P_{E}$ has determinant 1 .

Definition 7. The discriminant of the Hill equation is defined by:

$$
\Delta(E)=\operatorname{trace}\left(P_{E}\right) .
$$

The graph of $\Delta(E)$ looks like Fig. 15 .

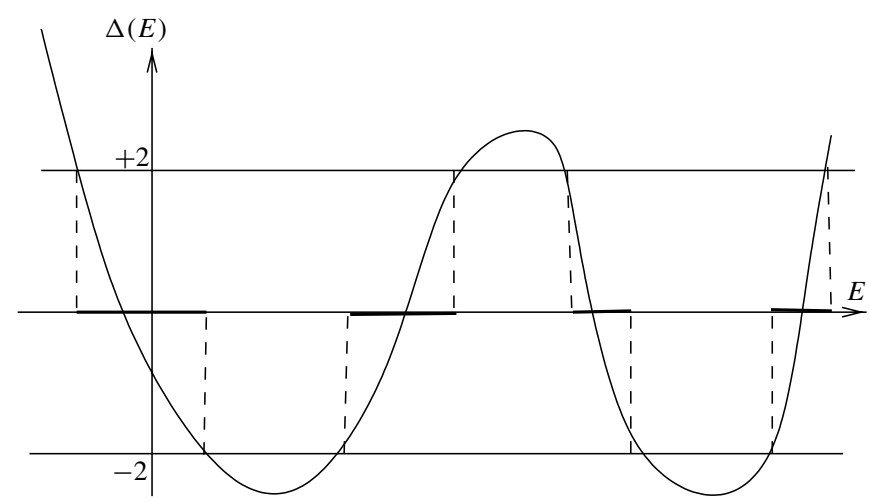

Fig. 15. The discriminant $\Delta(E)$

We define the periodic spectrum $E_{1}^{+}<\cdots \leq E_{k}^{+} \leq \cdots$ as the spectrum of the operator $\hat{H}$ restricted to the periodic functions. The $E_{k}^{+}$'s are precisely the solutions of $\Delta\left(E^{+}\right)=2$. We have a similar definition for the anti-periodic spectrum $E_{1}^{-} \leq \cdots \leq$ $E_{k}^{-} \leq \cdots$, this is the spectrum of $\hat{H}$ restricted to anti-periodic functions. The $E_{k}^{-}$'s are the solutions of $\Delta\left(E^{-}\right)=-2$. The following inequalities hold:

$$
E_{1}^{+}<E_{1}^{-} \leq E_{2}^{-}<E_{2}^{+} \leq E_{3}^{+}<E_{3}^{-} \leq \cdots
$$


and the bands are given by:

$$
\begin{aligned}
B_{2 j-1} & =\left[E_{2 j-1}^{+}, E_{2 j-1}^{-}\right], j=1,2, \cdots, \\
B_{2 j} & =\left[E_{2 j}^{-}, E_{2 j}^{+}\right], j=1,2, \cdots .
\end{aligned}
$$

More generally, the spectrum of $\hat{H}$ over $k T$-periodic functions is the union of the solutions of: $\Delta(E)=2 \cos \frac{2 \pi j}{k}$.

The sizes of the bands are exponentially small for $E<0$ as $h \rightarrow 0^{+}$and the sizes of the gaps are exponentially small for $E>0$. We want to describe the transition between this two regions, for this we will compute the asymptotic expansion series of $\Delta_{1}(\lambda)=\Delta(h \lambda)$. Moreover, we will use this asymptotic to describe the spectrum of $H$ restricted to $k T$-periodic functions.

10.2. Semi-classical asymptotic of the discriminant if the potential has a unique maximum per period. In this section, we assume that $V^{-1}(0)=2 \pi \mathbb{Z}$ and $V^{\prime \prime}(0)<0$, e.g. $V(x)=\cos x-1$. Let $\gamma_{ \pm}$denote the 2 cycles of $T^{\star}\left(\frac{\mathbb{R}}{2 \pi \mathbb{Z}}\right)$ defined by $\gamma_{+}(s)=$ $(s, \sqrt{-2 V(s)}), 0 \leq s \leq 2 \pi$ and $\gamma_{-}(s)=(2 \pi-s,-\sqrt{-2 V(2 \pi-s)}), 0 \leq s \leq 2 \pi$.

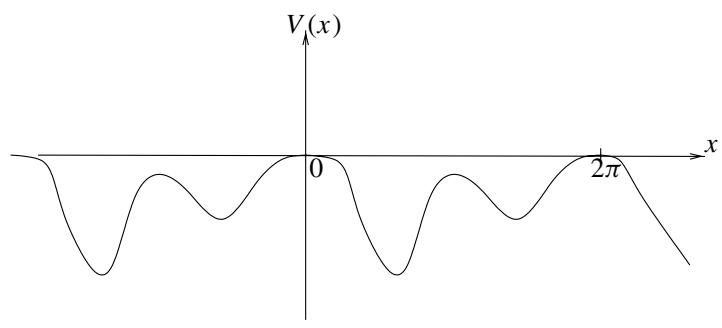

Fig. 16. An example of periodic potential

Theorem 7. The holonomies of $\gamma_{+}$and $\gamma_{-}$are equal. Let $\alpha$ be their common value:

$$
\alpha=e^{\frac{i \Omega}{h}+i \lambda J}+O(h),
$$

where $\Omega=\int_{\gamma_{+}} \xi d x$ and $J=\operatorname{vp} \int_{\gamma_{+}} d t$. Let $\varepsilon$ be the semi-classical invariant of the critical point $(0,0)$ :

$$
\varepsilon=\sum_{j=0}^{\infty} \varepsilon_{j}(\lambda) h^{j}, \quad \varepsilon_{0}=\frac{\lambda}{\left|V^{\prime \prime}(0)\right|^{\frac{1}{2}}} .
$$

The following relation holds for $|\lambda| \leq M$ :

$$
\Delta_{1}(\lambda)=2 \sqrt{1+e^{-2 \pi \varepsilon}} \cos \left(-\arg \alpha+\varepsilon \ln h+\arg \Gamma\left(\frac{1}{2}+i \varepsilon\right)\right)+O\left(h^{\infty}\right),
$$

hence

$$
\Delta_{1}(\lambda)=2 \sqrt{1+e^{-2 \pi \varepsilon_{0}}} \cos \left(-\frac{\Omega}{h}-\lambda J+\varepsilon_{0} \ln h+\arg \Gamma\left(\frac{1}{2}+i \varepsilon_{0}\right)\right)+O(h \ln h) .
$$


This result extends to the $C^{\infty}$ case the result of März ([19]) which was valid under analyticity assumptions.

Corollary 1. It follows immediately that in the region where $\lambda \rightarrow 0$, we have:

$$
\Delta_{1}(\lambda)=2 \sqrt{2} \cos \left(\frac{\Omega}{h}+\frac{\lambda \ln h}{\left|V^{\prime \prime}(0)\right|^{\frac{1}{2}}}\right)+O(\lambda)+O(h \ln h) .
$$

Hence the gaps and bands have asymptotically the same length l:

$$
l=\frac{\pi h\left|V^{\prime \prime}(0)\right|^{\frac{1}{2}}}{2|\ln h|}
$$

Proof. Near $x=0^{+}$, a solution of the Schrödinger equation is characterized by its coordinates $\left.\left(x_{1}, x_{2}, x_{3}, x_{4}\right)\right)$ in the basis $\left(\phi_{j}\right)$ of microlocal solutions. Let $\left(y_{1}, \cdots, y_{4}\right)$ denote the coordinates of the same solution near $x=2 \pi$ with respect to the basis $\left(\psi_{j}\right)$ The $P_{E}$ matrix is conjuguated to the matrix defined by $\left(y_{3}, y_{2}\right)=\tilde{P}_{E}\left(x_{3}, x_{2}\right)$. Its expression is easily deduced from the $T$ matrix and the holonomies:

$$
y_{3}=\alpha x_{1}, x_{4}=\alpha y_{2}, \quad\left(\begin{array}{l}
x_{3} \\
x_{4}
\end{array}\right)=T(\varepsilon)\left(\begin{array}{l}
x_{1} \\
x_{2}
\end{array}\right) .
$$

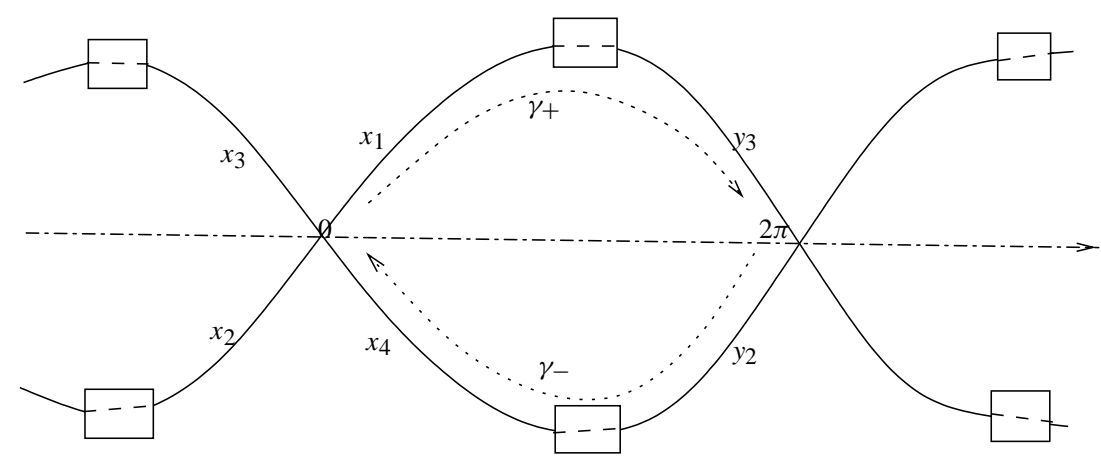

Fig. 17. The phase portrait for the Hill's equation

Factoring $\mathcal{E}$ in the $T(\varepsilon)$ matrix (cf. (18)), we get:

$$
\tilde{P}_{E}=\left(\begin{array}{ll}
\frac{\alpha}{\mathcal{E}} & -i \alpha e^{-\pi \varepsilon} \\
\frac{i e^{-\pi \varepsilon}}{\alpha} & \frac{\mathcal{E}\left(1+e^{-2 \pi \varepsilon}\right)}{\alpha}
\end{array}\right)
$$

Hence $\operatorname{det}\left(P_{E}\right)=1$ as it should be and:

$$
\operatorname{tr}\left(P_{E}\right)=2 \sqrt{1+e^{-2 \pi \varepsilon}} \cos \left(-\arg (\alpha)+\varepsilon \ln (h)+\arg \Gamma\left(\frac{1}{2}+i \varepsilon\right)\right) .
$$


10.3. The periodic spectrum. The periodic spectrum is easily deduced from the equation $\Delta_{1}(\lambda)=2$. We will now determine it by a direct method. We will also describe these two methods in Sect. 11 in the more complicated case of the spectrum of the Hill's equation on $2 T$ and $3 T$ periodic functions. The situation is similar to the double well potential, but here the pairs of exponentially close eigenvalues occur for energy value that are greater (instead of smaller) than the critical energy, these pairs come from the microlocal effect between the two classical symmetric trajectories.

In the transition energy interval, one has (cf. Fig. 18):

$$
x_{3}=\alpha x_{1}, x_{4}=\alpha x_{2} \quad\left(\begin{array}{l}
x_{3} \\
x_{4}
\end{array}\right)=T(\varepsilon)\left(\begin{array}{l}
x_{1} \\
x_{2}
\end{array}\right) .
$$

Hence 1 is eigenvalue of the unitary matrix:

$$
\left(\begin{array}{ll}
a & b \\
c & d
\end{array}\right):=T(\varepsilon)\left(\begin{array}{ll}
\alpha & 0 \\
0 & \alpha
\end{array}\right)
$$

As for the double-well potential, we apply the following equation from [8, Appendix]:

$$
|a| \cos \left(\frac{\arg a d}{2}-\arg a\right)=\cos \left(\frac{\arg a d}{2}\right)
$$

and we get the singular Bohr-Sommerfeld quantization rules:

$$
\frac{1}{\sqrt{1+e^{-2 \pi \varepsilon}}}=\cos \left(-\arg \alpha+\varepsilon \ln h+\arg \Gamma\left(\frac{1}{2}+i \varepsilon\right)\right) .
$$

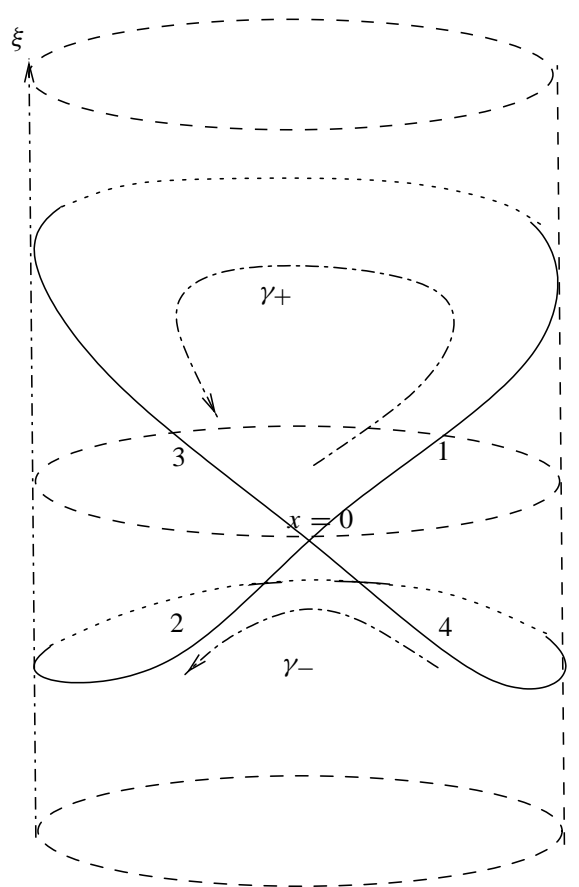

Fig. 18. The 0 energy trajectory in the phase space for the pendulum 
This equation may be deduced from (48) and $\Delta_{1}(\lambda)=2$ as well. Let us remark the minus sign in front of $\varepsilon$ in the exponential of the left-hand side of (54); it confirms the existence of pairs of eigenvalues for $E>0$ instead of $E<0$ (as obtained for the symmetric double-well potential). A more precise study of the solutions of (54) could be done, and would show the same universal transition of the eigenvalue ladders.

Example. For the Mathieu Equation $V(x)=\cos x-1$, we find $\Omega=8$ and $J=-5 \ln 2$. We have done a numerical comparison of the eigenvalues obtained by applying the singular Bohr-Sommerfeld rules and by using direct numerical methods, see Sect. A.2.

10.4. The semi-classical asymptotic of the discriminant for two maxima per period. In this section, we study the Hill's equation assuming that the potential has two maxima per period. With the notations of Fig. 19, it suffices to calculate $\left(B^{+}, Y\right)$ as a function of $\left(b^{+}, y\right)$. The holonomy equations read:
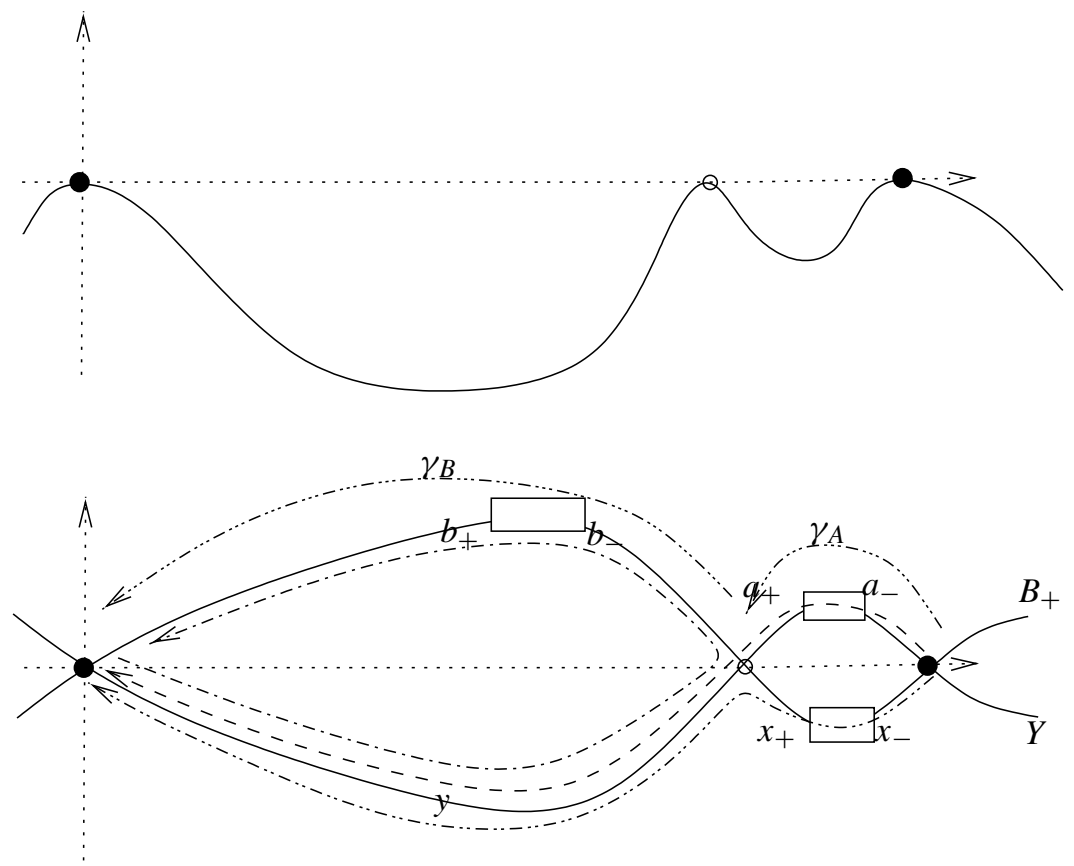

Fig. 19. The double well for the pendulum

$$
a^{+}=a^{-\frac{\alpha}{\beta}}, \quad b^{+}=b^{-} \beta^{2}, \quad x^{+}=\frac{x^{-}}{\alpha \beta}
$$

with $\alpha=\operatorname{hol}\left(\gamma_{A}\right)$ and $\beta=\operatorname{hol}\left(\gamma_{B}\right)$, and the critical invariant equations are:

$$
\left(\begin{array}{c}
b^{-} \\
x^{+}
\end{array}\right)=T(\eta)\left(\begin{array}{c}
a^{+} \\
y
\end{array}\right), \quad\left(\begin{array}{c}
a^{-} \\
Y
\end{array}\right)=T(\varepsilon)\left(\begin{array}{c}
B^{+} \\
x^{-}
\end{array}\right) .
$$


Using again our preferred CAS, we determine the function $\left(b^{+}, y\right) \rightarrow\left(B^{+}, Y\right)$. We check that the corresponding matrix has determinant 1 , and its trace is given by:

$$
\begin{aligned}
\Delta= & 2 \sqrt{1+e^{-2 \pi \varepsilon}} \sqrt{1+e^{-2 \pi \eta}} \cos (\arg \alpha+\arg \beta+\arg \mathcal{E}+\arg \mathcal{N})+ \\
& +2 e^{-\pi(\varepsilon+\eta)} \cos (\arg \beta-\arg \alpha) .
\end{aligned}
$$

It is now easy to get information about the lengths of the bands and gaps as $\lambda$ tends to 0 . Modulo a relative error of order $1 /|\ln (h)|$, we get:

$$
\Delta=4 \cos (\arg \alpha+\arg \beta+(\varepsilon+\eta) \ln (h))+2 \cos (\arg \beta-\arg \alpha),
$$

and we rewrite this equation as:

$$
\Delta=2+4\left[\cos (\arg \alpha+\arg \beta+(\varepsilon+\eta) \ln (h))-\sin ^{2}\left(\frac{\arg \beta-\arg \alpha}{2}\right)\right] .
$$

Therefore the inequality $\Delta>2$ is equivalent to:

$$
\cos (\arg \alpha+\arg \beta+(\varepsilon+\eta) \ln (h))>\sin ^{2}\left(\frac{\arg \beta-\arg \alpha}{2}\right)
$$

up to a $1 /|\ln (h)|$ relative error. Hence, the bands and gaps do not have generically the same length near the 0 energy, except if $\beta=\alpha$. These lengths depend on the value of $\arg \beta-\arg \alpha$. For example it is possible to have gaps of asymptotically small length or, conversely, bands of asymptotically small length.

\section{The Periodic Spectrum Over $2 T$ and $3 T$ Periodic Functions}

We proved in the previous section that the pendulum showed the same universal transition as the double well potential. It is natural to explore the situation of the double and triple translation-symmetric well over $M=\mathbb{R} /(2 \pi \mathbb{Z})$. The reference models are $V(x)=$ $1-\cos (2 x)$ and $1-\cos (3 x)$.

\subsection{The translation-symmetric double well.}

11.1.1. Direct method. In this situation, we will have pairs of exponentially close eigenvalues in both energy domains ( $E>0$ and $E<0$ ), but the tunneling trajectories that explain these couplings are different.

We can keep the notations of Fig. 19 but we have now $\alpha=\beta$ : there is a little cycle of holonomy $\alpha^{2}$, and two big cycles of holonomies $\alpha^{-2}$ and 1 . The two critical points have the same semi-classical invariant $\varepsilon$.

We get:

$$
\left(\begin{array}{c}
a^{-} \\
y \\
b^{-} \\
x^{+}
\end{array}\right)=\left(\begin{array}{cc}
T(\varepsilon) & 0_{2} \\
0_{2} & T(\varepsilon)
\end{array}\right)\left(\begin{array}{c}
b^{+} \\
x^{-} \\
a^{+} \\
y
\end{array}\right)=\left(\begin{array}{cccc}
0 & 0 & 1 & 0 \\
0 & 0 & 0 & 1 \\
\alpha^{-2} & 0 & 0 & 0 \\
0 & \alpha^{-2} & 0 & 0
\end{array}\right)\left(\begin{array}{c}
b^{+} \\
x^{-} \\
a^{+} \\
y
\end{array}\right) .
$$


This homogeneous system has a non-trivial solution $\left(b^{+}, x^{-}, a^{+}, y\right)$ if and only if its determinant is 0 . Factoring $\mathcal{E}$ in each line (cf. (18)) gives:

$$
\left|\begin{array}{llll}
1 & i e^{-\varepsilon \pi} & -1 / \mathcal{E} & 0 \\
i e^{-\varepsilon \pi} & 1 & 0 & -1 / \mathcal{E} \\
-1 / \mathcal{E} \alpha^{2} & 0 & 1 & i e^{-\varepsilon \pi} \\
0 & -1 / \mathcal{E} \alpha^{2} & i e^{-\varepsilon \pi} & 1
\end{array}\right|=0
$$

and eventually:

$$
\mathcal{E}\left(1-i e^{-\varepsilon \pi}\right)= \pm \frac{1}{\alpha} \text { where } \mathcal{E}\left(1+i e^{-\varepsilon \pi}\right)= \pm \frac{1}{\alpha} .
$$

As a consequence, we obtain the equidistribution of the eigenvalues near energy 0 in the semi-classical limit (here "near" means after a zoom-out of factor $1 / h$ ).

11.1.2. The trace method. The eigenvalues may be distinguished by parity properties: the periodic [respectively anti-periodic] eigenvalues correspond to eigenfunctions that satisfy $f(x+\pi)=f(x)$ [resp. $f(x+\pi)=-f(x)$ ]. Hence we have to check if 1 and -1 are eigenvalues of the translation matrix over a half period, or, since the determinant of this matrix is 1 , to check that the trace is 2 (periodic eigenvalue) or -2 (anti-periodic). Therefore we are lead to the equation:

$$
\cos \left(-\arg (\alpha)+\varepsilon \ln (h)+\arg \Gamma\left(\frac{1}{2}+i \varepsilon\right)\right)= \pm \frac{1}{\sqrt{1+e^{-2 \pi \varepsilon}}} .
$$

For $\varepsilon=0$, we find again the equidistribution of the eigenvalues with the following parity: periodic, anti-periodic, anti-periodic, periodic.

We can compare (56) and (55): since all members of (55) have modulus 1, these 4 equations are equivalent to the equations obtained by taking the arguments. Getting (56) is now easy.

Let us apply Eq. (10.4) with $\alpha=\beta$ and $\varepsilon=\eta$. Observing that $\cos (2 x)=2 \cos ^{2} x-1$ and that the orientation convention of the cycles are reversed to those of (47) (hence holonomies are inverses), we eventually get (56).

\subsection{The translation-symmetric triple well.}

11.2.1. Direct method. The critical energy area makes here a transition between triplets $(E<0)$ and pairs $(E>0)$ of exponentially close eigenvalues.

The semi-classical invariants of this situation are:

- two "small" cycles having the same holonomy denoted by $\alpha^{2}$,

- two "big" cycles, their holonomies are $\alpha^{-1}$ and $\alpha^{-3}=a^{3}$,

- three vertices having the same invariant $\varepsilon$.

With the notations of Fig. 20, we get:

$$
\left(\begin{array}{c}
a^{-} \\
z \\
b^{-} \\
x^{+} \\
c^{-} \\
y
\end{array}\right)=\left(\begin{array}{ccc}
T(\varepsilon) & 0 & 0 \\
0 & T(\varepsilon) & 0 \\
0 & 0 & T(\varepsilon)
\end{array}\right)\left(\begin{array}{c}
c^{+} \\
x^{-} \\
a^{+} \\
y \\
b^{+} \\
z
\end{array}\right)=\left(\begin{array}{cccccc}
0 & 0 & \alpha & 0 & 0 & 0 \\
0 & 0 & 0 & 0 & 0 & 1 \\
0 & 0 & 0 & 0 & \alpha^{-2} & 0 \\
0 & \alpha^{-3} & 0 & 0 & 0 & 0 \\
\alpha^{-2} & 0 & 0 & 0 & 0 & 0 \\
0 & 0 & 0 & 1 & 0 & 0
\end{array}\right)\left(\begin{array}{c}
c^{+} \\
x^{-} \\
a^{+} \\
y \\
b^{+} \\
z
\end{array}\right) .
$$




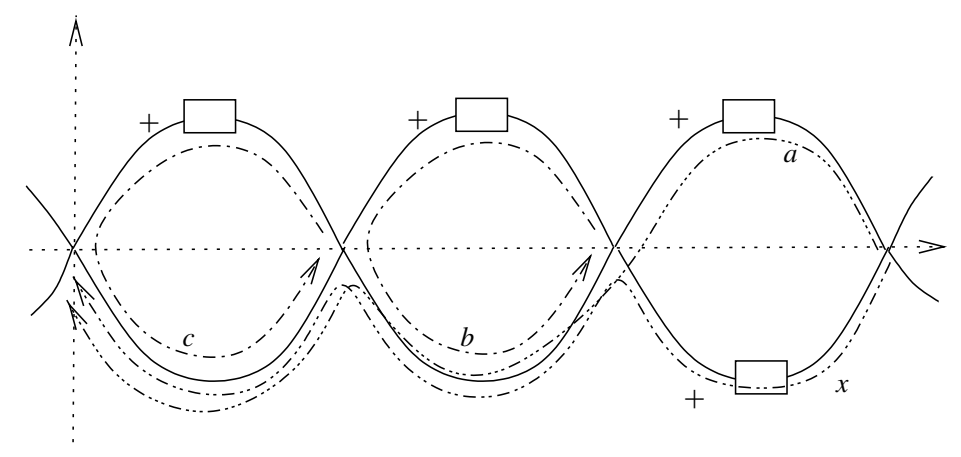

Fig. 20. The translation-symmetric triple well potential

Factoring $\mathcal{E}$ in the $T(\varepsilon)$ matrix (see (18)), we get the quantization rule as the nullity of the determinant of a $6 \times 6$ matrix:

$$
\left|\begin{array}{llllll}
1 & i e^{-\pi \varepsilon} & -\alpha / \mathcal{E} & 0 & 0 & 0 \\
i e^{-\pi \varepsilon} & 1 & 0 & 0 & 0 & -1 / \mathcal{E} \\
0 & 0 & 1 & i e^{-\pi \varepsilon}-1 / \alpha^{2} \mathcal{E} & 0 \\
0 & -1 / \alpha^{3} \mathcal{E} & i e^{-\pi \varepsilon} & 1 & 0 & 0 \\
-1 / \alpha^{2} \mathcal{E} & 0 & 0 & 0 & 1 & i e^{-\pi \varepsilon} \\
0 & 0 & 0 & -1 / \mathcal{E} & i e^{-\pi \varepsilon} & 1
\end{array}\right|=0 .
$$

This determinant can be factored as:

$$
\left(1-\alpha \mathcal{E}\left(1-i e^{-\pi \varepsilon}\right)\right)\left(1-\alpha \mathcal{E}\left(1+i e^{-\pi \varepsilon}\right)\right)\left(1+\alpha \mathcal{E}+\alpha^{2} \mathcal{E}^{2}\left(1+e^{-2 \pi \varepsilon}\right)\right)^{2}=0 .
$$

Hence we find two simple eigenvalues, solutions of:

$$
\mathcal{E}\left(1-i e^{-\pi \varepsilon}\right)= \pm \frac{1}{\alpha}
$$

and two eigenvalues of multiplicity 2 , solutions of:

$$
\mathcal{E}=\frac{-1 \pm i \sqrt{4\left(1+e^{-2 \pi \varepsilon}\right)-1}}{2 \alpha\left(1+e^{-2 \pi \varepsilon}\right)} .
$$

If we make a zoom-out of the eigenvalues near 0 , their repartition will be asymptotically the same as the repartition of the following complexes on the unit circle:

$$
\frac{-1 \pm i}{\sqrt{2}}(\text { simple }), \quad \frac{-1 \pm i \sqrt{7}}{2 \sqrt{2}}(\text { double })
$$

Therefore the transition area looks like Fig. 21 where we have used a bold level for double eigenvalues on the ladder. 


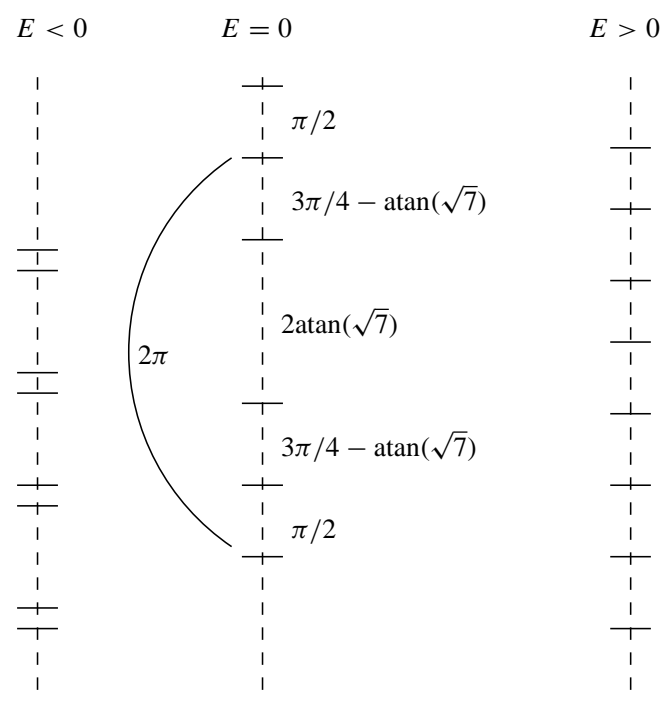

Fig. 21. Universal transition for the triple well

11.2.2. The trace method. We study the trace of the translation matrix on one third of a period. The periodicity/anti-periodicity of eigenfunctions is replaced by the relation:

$$
f\left(x+\frac{2 \pi}{3}\right)=e^{2 i k \pi / 3} f(x)
$$

Remark that $k=1$ and $k=2$ correspond to eigenfunctions that are conjugated, this explains the fact that eigenvalues of multiplicity 2 correspond to a trace of $2 \cos (2 \pi / 3)$. The non degenerate eigenvalues correspond to eigenfunctions that are $2 \pi / 3$ periodic, the transition of these eigenvalues will therefore be similar to the transition of the eigenvalues of the single pendulum. For multiplicity 2 eigenvalues, we get:

$$
\cos \left(-\arg (\alpha)+\varepsilon \ln (h)+\arg \Gamma\left(\frac{1}{2}+i \varepsilon\right)\right)= \pm \frac{1}{2 \sqrt{1+e^{-2 \pi \varepsilon}}} .
$$

As an easy consequence, we obtain for $\varepsilon=0$ the same description as in Fig. 21.

\section{System of Schrödinger Operators with a Small Coupling Parameter}

This section is related to [21] and [6], see also [5]. Let $\hat{P}_{j}, j=1,2$ be two Schrödinger operators with potentials $V_{j}(x)$ defined by $\hat{P}_{j}=-\frac{h^{2}}{2} \frac{d^{2}}{d x^{2}}+V_{j}(x)$ with smooth $V_{j}$ 's. Assume that the characteristic manifolds $Z_{j}=\left\{\frac{1}{2} \xi^{2}+V_{j}(x)=0\right\}$ are smooth circles intersecting transversally at the points $z_{ \pm}=\left(x_{0}, \pm \xi_{0}\right)$. We are interested to study existence of microlocal solutions for the system:

$$
\begin{aligned}
& \hat{P}_{1} u+\varepsilon v=0, \\
& \varepsilon u+\hat{P}_{2} v=0 .
\end{aligned}
$$


If $\varepsilon=0$, we get two independent equations. If $\varepsilon \neq 0, u$ is solution of the fourth order equation

$$
\hat{H} u=\left(\hat{P}_{2} \hat{P}_{1}-\varepsilon^{2}\right) u=0 .
$$

If $\varepsilon \neq 0$ is small and independent of $h$ we get a smooth characteristic manifold. If we assume $\varepsilon=O(\sqrt{h})$, we get a singular case which can be studied using the previous tools. It is then natural to put $\varepsilon=\omega \sqrt{h}$ with $\omega \neq 0$. Let us denote by $T_{ \pm}$, the matrices

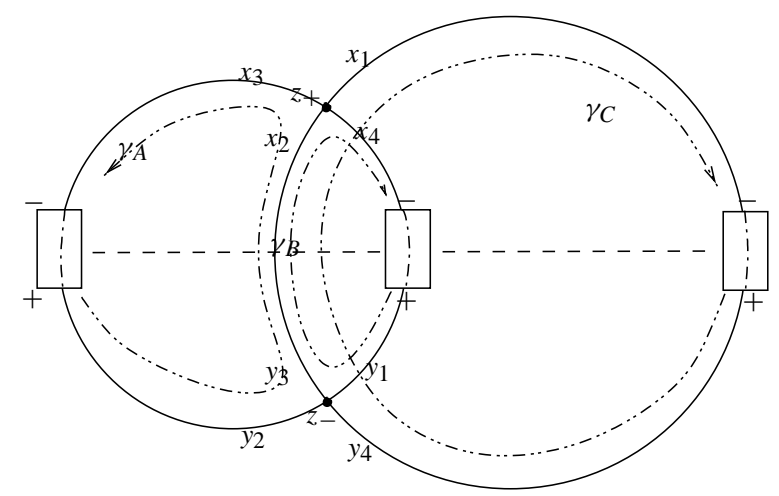

Fig. 22. Phase space for $\hat{H}$

associated with the singular points $z_{ \pm}$. If $\gamma_{A}, \gamma_{B}$ and $\gamma_{C}$ are the cycles defined by Fig. 22 and $\alpha=\operatorname{hol}\left(\gamma_{A}\right), \beta=\cdots$, we have the following equations:

$$
\begin{aligned}
& \left(\begin{array}{l}
x_{3} \\
x_{4}
\end{array}\right)=T_{+}\left(\begin{array}{l}
x_{1} \\
x_{2}
\end{array}\right),\left(\begin{array}{l}
y_{3} \\
y_{4}
\end{array}\right)=T_{-}\left(\begin{array}{l}
y_{1} \\
y_{2}
\end{array}\right), \\
& x_{2}=y_{3}, y_{4}=\gamma x_{1}, y_{1}=\beta x_{4}, y_{2}=\alpha x_{3} .
\end{aligned}
$$

Hence, the singular Bohr-Sommerfeld rule reads:

$$
\operatorname{det}\left(\operatorname{Id}-T_{+}\left(\begin{array}{cc}
0 & \gamma^{-1} \\
1 & 0
\end{array}\right) T_{-}\left(\begin{array}{cc}
0 & \beta \\
\alpha & 0
\end{array}\right)\right)=0
$$

Let us sketch the way to compute the holonomies and $\varepsilon_{0}$ 's of singular points.

We have $H_{0}=\left(\frac{1}{2} \xi^{2}+V_{2}(x)\right)\left(\frac{1}{2} \xi^{2}+V_{1}(x)\right)$ and $H_{1}=\frac{1}{2 i} \xi\left(V_{2}^{\prime}-V_{1}^{\prime}\right)(x)-\omega^{2}$. We get

$$
\varepsilon_{0}\left(z_{ \pm}\right)=\mp \frac{1}{2 i}+\frac{\omega^{2}}{\left|\xi_{0}\left(V_{2}^{\prime}-V_{1}^{\prime}\right)\left(x_{0}\right)\right|} .
$$

The matrices $T_{ \pm}$are not unitary because $H_{1}$ is no longer real, but it is possible to check that

$$
T_{+}\left(\begin{array}{cc}
0 & \gamma^{-1} \\
1 & 0
\end{array}\right) T_{-}\left(\begin{array}{cc}
0 & \beta \\
\alpha & 0
\end{array}\right)
$$

belongs to $U(1,1)$. 


\section{Scattering Matrices}

13.1. One channel scattering. We are interested in an example studied by Rouleux ([23]). Let $V(x)$ be a $C^{\infty}$ potential such that (see Fig. 23):

- $V(x)=0$ for $x \geq b>0$.

- $V(0)=V_{0}>0, V^{\prime}(0)=0$ and $V^{\prime \prime}(0)<0$.

- $V^{-1}\left(V_{0}\right)=\{a, 0\}$ for an $a<0$ such that $V^{\prime}(a)<0$.

Hence $V^{-1}\left(\left(-\infty, V_{0}\right]\right)=[a,+\infty($.

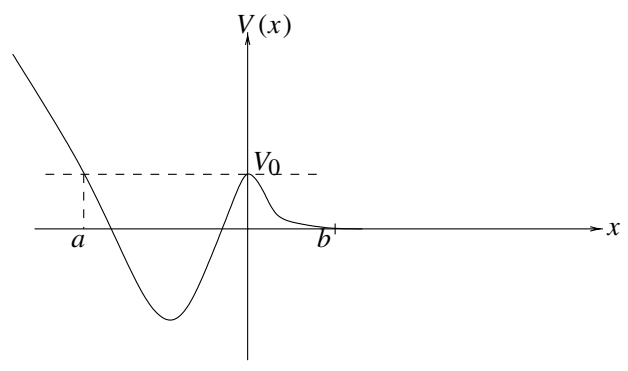

Fig. 23. 1 channel scattering potential

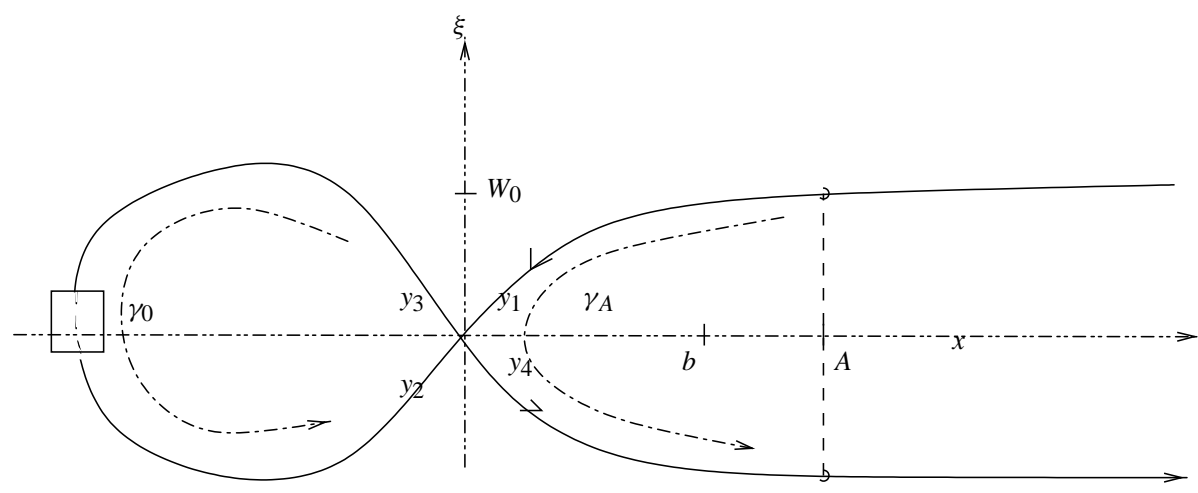

Fig. 24. 1 channel scattering in the phase space

Let $k>0$. The Schrödinger equation

$$
-\frac{h^{2}}{2} \varphi^{\prime \prime}+V(x) \varphi=k^{2} \varphi
$$

has a unique solution $\varphi \in L^{2}\left(\mathbb{R}^{-}\right)$, such that:

$$
\varphi=r(k) e^{i \sqrt{2} k x / h}+e^{-i \sqrt{2} k x / h}, \quad x \geq b .
$$

It is well known that $r(k)$ has modulus $1, r(k)$ is called the reflection coefficient. Using the previous methods, we will calculate $r(k)$ up to an error of order $O(h)$ for $k=$ $\sqrt{V_{0}}+O(h)$. 
Let $W_{o}=\sqrt{V_{0}}$. We may write $k=W_{0}+h \omega$ with $\omega=O(1)$. Hence, $H_{0}=$ $\frac{1}{2} \xi^{2}+V(x)-V_{0}$ and $H_{1}=-2 W_{0} \omega$.

Fix $A>b$ and let $\gamma_{A}$ be the simple path of the phase space joining $\left(A, W_{0}\right)$ to $\left(A,-W_{0}\right)$ inside the characteristic manifold $\frac{1}{2} \xi^{2}+V(x)=V_{0}$. The phase shift of a standard solution along $\gamma_{A}$ is given (see (32)) by:

$$
\varphi_{A}=\frac{1}{h} \int_{\gamma_{A}} \xi d x-\text { v.p. } \int_{\gamma_{A}} H_{1} d t+O(h) .
$$

If $e^{i \theta_{0}}$ is the holonomy of the loop $\gamma_{0}$ and, if we put $\Omega=2 \sqrt{2} k A+\varphi_{A}$ (which is independent of the choice of $A>b$ ), we get:

$$
y_{2}=e^{i \theta_{0}} y_{3}, \quad y_{1}=r(k) e^{i \Omega} y_{4}
$$

and

$$
\left(\begin{array}{l}
y_{3} \\
y_{4}
\end{array}\right)=T(\varepsilon)\left(\begin{array}{l}
y_{1} \\
y_{2}
\end{array}\right)
$$

Hence $r(k)=e^{i \Phi}$ with:

$$
\Phi=-\left(\Omega+\theta_{0}+2 \arg (\mathcal{E})\right)+2 \arg \left(1-i e^{-\pi \varepsilon} \mathcal{E} e^{i \theta_{0}}\right)+O(h) .
$$

13.2. Two channel scattering. Let us consider the volcano-top example. This is a Schrödinger equation on $\mathbb{R}$ like (33) with the following assumptions:

- $V(x) \rightarrow V_{-}, V_{-}<0$ as $x \rightarrow+\infty$,

- $V(x) \rightarrow V_{+}, V_{+}<0$ as $x \rightarrow-\infty$,

- $\sup V=0$

- $V^{-1}(0)=\left\{x_{1}, x_{2}\right\}$ with $x_{1}<x_{2}$ and $V^{\prime \prime}\left(x_{i}\right)<0$.

This example has been studied by Fujiie and Ramond ([14]) in the analytic case.

The energy shell in phase space looks like Fig. 25. Let $\beta=e^{i \theta}$ be the holonomy of

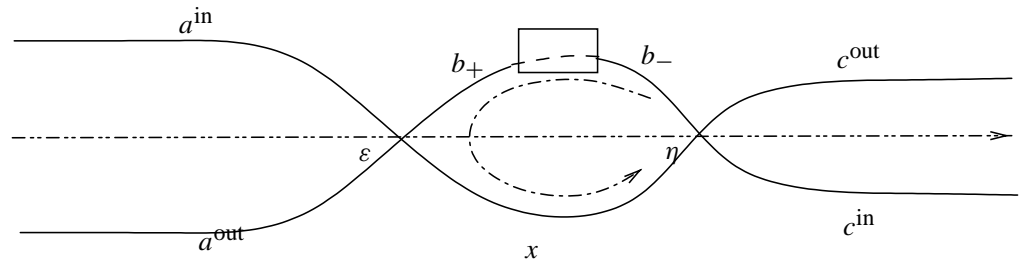

Fig. 25. The volcano-top

the cycle, and $\mathcal{E}_{j}, j=1,2$ be the scalar coefficients factored in the matrices $T\left(\varepsilon_{j}\right)$ (cf.

(18)). Then the following equations hold:

$$
b^{+}=\beta b_{-}, \quad\left(\begin{array}{c}
a^{\text {in }} \\
x
\end{array}\right)=T\left(\varepsilon_{1}\right)\left(\begin{array}{c}
b^{+} \\
a^{\text {out }}
\end{array}\right), \quad\left(\begin{array}{c}
b_{-} \\
c^{\text {in }}
\end{array}\right)=T\left(\varepsilon_{2}\right)\left(\begin{array}{c}
c^{\text {out }} \\
x
\end{array}\right) .
$$


By Gaussian elimination of $b$ and $x$, we get:

$$
\begin{aligned}
a^{\text {in }} & =\frac{\mathcal{E}_{1}}{D}\left[i\left(\beta \mathcal{E}_{1} \mathcal{E}_{2} e^{-\varepsilon_{2} \pi}\left(1+e^{-2 \varepsilon_{1} \pi}\right)+e^{-\varepsilon_{1} \pi}\right) a^{\text {out }}+\beta \mathcal{E}_{2} c^{\text {out }}\right], \\
c^{\text {in }} & =\frac{\mathcal{E}_{2}}{D}\left[\mathcal{E}_{1} a^{\text {out }}+i\left(\beta \mathcal{E}_{1} \mathcal{E}_{2} e^{-\varepsilon_{1} \pi}\left(1+e^{-2 \varepsilon_{2} \pi}\right)+e^{-\varepsilon_{2} \pi}\right) c^{\text {out }}\right], \\
D & =1+e^{-\varepsilon_{1} \pi} e^{-\varepsilon_{2} \pi} \beta \mathcal{E}_{1} \mathcal{E}_{2} .
\end{aligned}
$$

Note that the denominator $D$ is never 0 for real energies since $\left|\mathcal{E}_{j}\right|=\left(1+e^{-2 \varepsilon_{j} \pi}\right)^{-1 / 2}$. The complex zeroes of the analytic continuation of $D$ are named quasi-resonances. We remark that the $S$ matrix such that $S\left(a^{\text {out }}, c^{\text {out }}\right)=\left(a^{\text {in }}, c^{\text {in }}\right)$ is unitary as it should.

We will now calculate the modulus of the transmission and reflection coefficients (it is of course possible to calculate the phase shifts in the spirit of the previous section). Let us consider a solution which is micro-locally supported on one of the two incoming branches, e.g. $a^{\text {in }}$. Hence $a^{\text {in }}=1, c^{\text {in }}=0$ and $\left|c^{\text {out }}\right|=|t|,\left|a^{\text {out }}\right|=|r|=\sqrt{1-|t|^{2}}$. Since $S$ is unitary, we have:

$$
|t|=\left|\frac{\mathcal{E}_{2} \mathcal{E}_{1}}{D}\right|
$$

For example, if we are interested in energies $E$ such that $E / h$ tends to 0 , then $\varepsilon_{1}=\varepsilon_{2}=0$ and we find the same result as Fujiie and Ramond ([14]):

$$
|t|=\frac{1}{\sqrt{5+4 \cos \theta}} .
$$

\section{A. Numerical Results}

A.1. The symmetric double well potential. Let $V(x)=x^{4}-x^{2}$. In this section, we calculate the eigenvalues of the corresponding Schrödinger operator for different values of $h$ by solving (41) or by applying the Runge-Kutta method to the first order differential system:

$$
\frac{d}{d x}\left(\begin{array}{c}
\varphi \\
\varphi^{\prime}
\end{array}\right)=\left(\begin{array}{cc}
0 & 1 \\
\frac{2(V(x)-E)}{h^{2}} & 0
\end{array}\right) \cdot\left(\begin{array}{c}
\varphi \\
\varphi^{\prime}
\end{array}\right)
$$

More precisely, we calculate two solutions of (59), starting from two points in the classically forbidden region, the first one is chosen in the connected component of $-\infty$ and the second in the component of $+\infty$. We take $\varphi=1$ and $\varphi^{\prime}=0$ as initial conditions at these points. And we compute the Wronskian $W(E)$ of these 2 solutions at $x=0$. Since we start from the classically forbidden region, the numerical solution that we obtain in the classically allowed region will behave like a solution which decreases exponentially fast in the component of the starting point region. Therefore, the Wronskian $W(E)$ will vanish if and only if $E$ is an eigenvalue. To find these values, we detect sign changes of $W(E)$ first by dichotomy, then by interpolation of the curve $E \rightarrow W(E)$ for better accuracy (this is the best method when Newton's method can not be applied. The convergence law of the sequence of $E$ is given by $\left|E_{n}-E\right| \leq\left|E_{0}-E\right|^{F_{n}}$ where $F_{n}$ are the Fibonacci numbers). 
On the other hand, rewriting Eq. (41) for the potential $V(x)=x^{4}-x^{2}$ ( $a=1$ and $b=0)$ gives:

$$
\frac{1}{\sqrt{1+e^{2 \pi \varepsilon}}}+\cos \left(\varepsilon \ln (h)+\arg \Gamma\left(\frac{1}{2}+i \varepsilon\right)-\frac{7}{2} \varepsilon \ln (2)-\frac{2 \sqrt{2}}{3 h}\right),
$$

where $\varepsilon=\frac{E}{\sqrt{2} h}$.

All these functions are standard, except the argument of the $\Gamma$ function. We have used the PARI library from the number theory laboratory of Bordeaux to calculate it. Implementation has been done in $\mathrm{C} / \mathrm{C}++$, programs are available at:

http: //www-fourier.ujf-grenoble.fr/parisse/bs.tgz

For $h=10^{-6}$, we get the following eigenvalues in the interval $\left[-2.10^{-6}, 3.10^{-6}\right]$ :

\begin{tabular}{lrr}
\hline Index & Runge-Kutta & Bohr-Sommerfeld \\
\hline 1 & $-1.68092 \mathrm{e}-06$ & $-1.68100 \mathrm{e}-06$ \\
2 & $-1.67670 \mathrm{e}-06$ & $-1.67678 \mathrm{e}-06$ \\
3 & $-1.14072 \mathrm{e}-06$ & $-1.14080 \mathrm{e}-06$ \\
4 & $-1.12697 \mathrm{e}-06$ & $-1.12706 \mathrm{e}-06$ \\
5 & $-0.62743 \mathrm{e}-06$ & $-0.62751 \mathrm{e}-06$ \\
6 & $-0.58579 \mathrm{e}-06$ & $-0.58587 \mathrm{e}-06$ \\
7 & $-0.15515 \mathrm{e}-06$ & $-0.15522 \mathrm{e}-06$ \\
8 & $-0.05032 \mathrm{e}-06$ & $-0.05041 \mathrm{e}-06$ \\
9 & $0.29793 \mathrm{e}-06$ & $0.29786 \mathrm{e}-06$ \\
10 & $0.48460 \mathrm{e}-06$ & $0.48451 \mathrm{e}-06$ \\
11 & $0.78346 \mathrm{e}-06$ & $0.78339 \mathrm{e}-06$ \\
12 & $1.02441 \mathrm{e}-06$ & $1.02432 \mathrm{e}-06$ \\
13 & $1.30685 \mathrm{e}-06$ & $1.30677 \mathrm{e}-06$ \\
14 & $1.57243 \mathrm{e}-06$ & $1.57234 \mathrm{e}-06$ \\
15 & $1.85275 \mathrm{e}-06$ & $1.85266 \mathrm{e}-06$ \\
16 & $2.12953 \mathrm{e}-06$ & $2.12944 \mathrm{e}-06$ \\
17 & $2.41218 \mathrm{e}-06$ & $2.41208 \mathrm{e}-06$ \\
18 & $2.69515 \mathrm{e}-06$ & $2.69506 \mathrm{e}-06$ \\
19 & $2.98110 \mathrm{e}-06$ & $2.98101 \mathrm{e}-06$ \\
20 & $3.26833 \mathrm{e}-06$ & $3.26824 \mathrm{e}-06$ \\
\hline
\end{tabular}

Note the accuracy of the singular Bohr-Sommerfeld rules. This method is far cheaper than the Runge-Kutta method (about $100 \times$ faster).

Comparison 2: For $h=0.1$, we get the following eigenvalues in the interval $[-0.3,0.3]$ :

\begin{tabular}{lrr}
\hline Index & Runge-Kutta & Bohr-Sommerfeld \\
\hline 0 & -0.156568 & -0.16038 \\
1 & -0.155061 & -0.15861 \\
2 & -0.014654 & -0.01358 \\
3 & 0.021032 & 0.02162 \\
4 & 0.116728 & 0.11546 \\
5 & 0.212051 & 0.20839 \\
\hline
\end{tabular}

Even for this relatively large $h$ value, the singular quantization rules give an accurate approximation of the eigenvalues. Note however that for this value of $h$, the Runge-Kutta 
method is faster! We remark that the mean gap between two consecutives eigenvalues is about $1 \times h$ in this example and about $4 \times h$ for $h=10^{-6}$. Theoretically, we should find a ratio of 6 instead of 4 . This would require to calculate eigenvalues for energies that are much closer to $E=0$.

Let us now compare the two methods (singular quantization rules and numerical integration) in terms of the position of the ladders of even and odd eigenvalues near the critical energy. We introduce the form factor:

$$
R=\frac{E_{2 n+1}-E_{2 n}}{E_{2 n+2}-E_{2 n}}
$$

where the eigenvalues are indexed starting from $n=0$ (hence even eigenvalues correspond to even eigenfunctions). For $E_{2 n+1}>>h$, eigenvalues are equidistributed, hence $R$ tends to $1 / 2$; for $-E_{2 n+1}>>h$, eigenvalues are coupled, hence $R$ tends to 0 . If $E_{2 n+1} /\left(h \sqrt{\left|V^{\prime \prime}(0)\right|}\right)$ tends to a limit $\varepsilon_{0}$ as $h$ tends to 0 , it is possible to calculate the limit of $R$ :

$$
\lim _{h \rightarrow 0} R=\frac{\arccos \left(\left(1+e^{2 \pi \varepsilon_{0}}\right)^{-1 / 2}\right)}{\pi} .
$$

Hence $R \rightarrow 1 / 4$ for $\varepsilon_{0}=0$. Coming back to the second example above ( $h=0.1$ ), we get $\left(E_{3}-E_{2}\right) /\left(E_{4}-E_{2}\right)=0.27$, a value which is close to the theoretical value of $R$ (since $E_{3} /(\sqrt{2} h)=0.15$ is close to 0$)$.

A.2. The pendulum. We make the same kind of comparison for the pendulum (the periodic Schrödinger equation), where we take $V(x)=\cos (x)-1$ as potential. The singular Bohr-Sommerfeld quantization rules read (see (54)):

$$
\frac{1}{\sqrt{1+e^{-2 \pi \varepsilon}}}=\cos \left(-\frac{8}{h}-5 \ln (2) \varepsilon+\varepsilon \ln h+\arg \Gamma\left(\frac{1}{2}+i \varepsilon\right)\right)
$$

with $\varepsilon=E / h$.

We use a numerical method which is slightly different than that of the previous section: here we will study the monodromy matrix $M$. Let $\left(\varphi, \varphi^{\prime}\right)=(1,0)$ and $\left(\varphi, \varphi^{\prime}\right)=(0,1)$ be a couple of (linearly independent) initial conditions at $x=0$. Using the Runge-Kutta method, we integrate the Schrödinger equation from $x=0$ to $x=2 \pi$ and determine the matrix $M$. Energy $E$ is an eigenvalue if and only if 1 is eigenvalue of $M$. We have now to find the zeroes of $\operatorname{det}(M-I)$.

Let $p$ be the length of a step of the Runge-Kutta method. Then the relative error is of order $p^{3} / h^{4}$ in the $E \geq 0$ energy area. The error is much bigger in the $E<0$ area because starting and ending in the classically forbidden region multiplies the relative error by an exponential coefficient which is proportional to $e^{-C E / h}$. Hence we can not apply this numerical method if $E<-D h$ (and if we choose a big value for $D$, we have to choose a correspondingly small value for $p$ ). 
The comparison shows that the two methods give close results. In the following example, we take $h=10^{-3}$, and we obtain:

\begin{tabular}{lll}
\hline Index & Runge-Kutta & Bohr-Sommerfeld \\
\hline 0 & -0.00339886 & -0.00339946 \\
1 & -0.00305773 & -0.00305821 \\
2 & -0.00272065 & -0.0027210 \\
3 & -0.00238812 & -0.00238841 \\
4 & -0.00206018 & -0.002060396 \\
5 & -0.00173847 & -0.001738624 \\
6 & -0.00142077 & -0.0014208684 \\
7 & -0.00111594 & -0.001116004 \\
8 & -0.000805647 & -0.000805677 \\
9 & -0.000541145 & -0.000541155 \\
10 & -0.000212948 & -0.000212945 \\
11 & $-5.18091 \mathrm{e}-05$ & $-5.18018 \mathrm{e}-05$ \\
12 & 0.000371877 & 0.0003718748 \\
13 & 0.000420734 & 0.000420730 \\
14 & 0.000978865 & 0.000978818 \\
15 & 0.000978865 & 0.000978818 \\
\hline
\end{tabular}

We note a better accuracy for $E>0$, as we could expect from the above remark about the instability of the numerical method for $E<0$.

\section{B. Proof of Theorem 2}

Isotropy is clear, because if $u$ is a solution in $U\left(u \in \mathcal{M}_{\hat{H}}(U)\right), \Sigma \hat{H} \Pi u=\hat{H} \Pi u$.

We only need to get a lower bound by induction on the number of compact edges. If there are no compact edges, we know already that the dimension of the space of solutions is half the number of open edges because in that case, $Z$ is an union of simple crosses. Gluing boundaries of a cut edge can be identified with a reduction on the $\mathcal{S}$-matrix. More precisely, we use the:

Lemma 3. Let $H=H_{+} \oplus H_{-}$, where $H_{+}$and $H_{-}$are 2 Hilbert spaces of the same finite dimension and $U: H_{-} \rightarrow H_{+}$be a linear isometry whose graph is $\Gamma . e_{ \pm} \in H_{ \pm}$ are 2 vectors of length 1 and $K_{ \pm}$are the orthogonals of $\mathbb{C} e_{ \pm}$. Let $e^{i \alpha}$ be a complex number of modulus 1 and

$$
\Gamma_{1}=\left\{\left(z_{-}, z_{+}\right) \in K_{-} \oplus K_{+} \mid \exists y \in \mathbb{C},\left(z_{-}+y e_{-}, z_{+}+e^{i \alpha} y e_{+}\right) \in \Gamma\right\} .
$$

Then $\Gamma_{1}$ is the graph of an unitary mapping from $K_{-}$into $K_{+}$.

The proof is elementary.

We then apply the lemma. Given $Z, Z_{1}$ is obtained by cutting some compact edge of $Z$ and replacing it by one in-going and one out-going edge of $Z_{1} . H_{ \pm}$are related to $Z_{1}$ and $K_{ \pm}$are related to $Z$. The functions $e_{ \pm}$are $J$-normalized microlocal solutions at both ends of the cut edge and $y_{+}=e^{i \alpha} y_{-}$expresses the gluing of both solutions. 


\section{References}

1. Bleher, P.: Semi-classical quantization rules near separatrices. Commun. Math. Phys. 165, 621-640 (1994)

2. Colin de Verdière, Y.: Formes normales de Birkhoff classiques et quantiques. Exposé au séminaire de physique-mathématique de l'Institut Fourier, 1997

3. Colin de Verdière, Y.: Quasi-modes sur les variétés riemanniennes. Inventiones Math. 43, 15-52 (1977)

4. Colin de Verdière, Y.: Spectre conjoint d'opérateurs pseudo-différentiels qui commutent II: le cas intégrable. Math. Zeitschrift 171, 51-73 (1980)

5. Colin de Verdière, Y.: Limite adiabatique en présence de croisements évités et phases géométriques. In progress, 1999

6. Colin de Verdière, Y., Lombardi, M. and Pollet, J.: La formule de Landau-Zener microlocale. Prépublication de l'Institut Fourier 412, 1-15 (1999)

(available at http://www-fourier.ujf-grenoble.fr/), to appear in Annales de l'IHP (Physique Théorique)

7. Colin de Verdière, Y.: and Parisse, B.: Equilibre instable en régime semi-classique: I-Concentration microlocale. Commun. PDE 19, 1535-1563 (1994)

8. Colin de Verdière, Y. and Parisse, B.: Equilibre instable en régime semi-classique: II-Conditions de BohrSommerfeld. Ann. Inst. Henri Poincaré (Physique théorique) 61, 347-367 (1994)

9. Colin de Verdière, Y. and Vey, J.: Le lemme de Morse isochore. Topology 18, 283-293 (1979)

10. Colin de Verdière, Y. and Vu Ngoc, S.: Singular Bohr-Sommerfeld Rules for $2 d$ Integrable Systems. In Progress 1999

11. Delabaere, E., Dillinger, H. and Pham, F.: Exact Semi-Classical Expansions For One Dimensional Quantum Oscillators. Journal math. Phys. 38, 6126-6184 (1997)

12. Delabaere, E. and Pham, F.: Unfolding the quartic oscillator. Annals of Phys. 261, 180-218 (1997)

13. Duistermaat, J.: Oscillatory integrals, Lagrange immersions and unfoldings of singularities. Commun. Pure appl. Math. 27, 207-281 (1974)

14. Fujiié, S. and Ramond, T.: Matrice de scattering et résonances associées à une orbite hétérocline. Annales de l'IHP, Physique Théorique 69 (1), 31-83 (1998)

15. Guillemin, V. and Schaeffer, D.: On a certain class of Fuchsian partial differential equations. Duke Math. J. 44, 157-199 (1977)

16. Joye, A.: Exponential asymptotics in a singular limit for $n$-level scattering systems. SIAM J. Math. Anal. 28, 669-703 (1997)

17. Joyeux, M.: Classical dynamics of the 1:1, 1:2 and 1:3 resonance Hamiltonians. J. Chem. Phys. 203, 281-307 (1996)

18. Martin, P. and Nenciu, G.: Semi-classical inelastic $S$-matrix for one dimensional $N$-states systems. Reviews in Math. Phys. 7, 193-242 (1995)

19. März, C.: Spectral asymptotics for Hill's equation near the potential maximum. Asymptotic Anal. 5, 221-267 (1992)

20. Maslov, V.P.: Théorie des perturbations et méthodes asymptotiques. Suivi de deux notes complémentaires de V. I. Arnol'd et V. C. Bouslaev. Paris: Dunod, Gauthier-Villars, 1972

21. Pollet, J.: Analyse semi-classique d'un système d'équations de Schrödinger couplées: Formule de LandauZener. Thèse de l'université de Grenoble, 1997

22. Robert, D.: Autour de l'Approximation Semi-Classique. Basel-Boston: Birkhäuser, 1987

23. Rouleux, M.: Tunneling for $h$-Pseudodifferential Operators, Feshbach Resonances and the BornOppenheimer Approximation. In: Evolution Equations, Feshbach resonances, Singular Hodge Theory, M. Demuth, E. Schrohe, B. Schultze (eds), Advances in PDE no. 16, New York: Wiley, 1999

24. Vu Ngoc, S.: Bohr-Sommerfeld conditions for Integrable Systems with critical manifold of focus-focus type. Prepublication de l'Institut Fourier no 433 (1998)

25. Vu Ngoc, S.: Sur le spectre des systèmes complètement intégrables semi-classiques avec singularités. $\mathrm{PhD}$ Thesis Grenoble, December 1998

26. Warner, F.: Foundations of Differentiable Manifolds and Lie Groups. Berlin-Heidelberg-New York: Springer, 1983

Communicated by B. Simon 